\title{
Holographic dual of a time machine
}

\author{
Irina Arefeva, ${ }^{1, *}$ Andrey Bagrov, ${ }^{2,3, \dagger}$ Petter Säterskog, ${ }^{2, \$}$ and Koenraad Schalm ${ }^{2, \S}$ \\ ${ }^{1}$ Steklov Mathematical Institute RAS, Moscow, Gubkina street 8, 119991 \\ ${ }^{2}$ Institute Lorentz, Leiden University, P.O. Box 9506, Leiden 2300 RA, The Netherlands \\ ${ }^{3}$ Institute for Molecules and Materials, Radboud University, \\ Heyendaalseweg 135, Nijmegen 6525 AJ, The Netherlands \\ (Received 5 September 2015; published 29 August 2016)
}

\begin{abstract}
Assuming that the AdS/CFT prescription is valid in the case of noncausal backgrounds, we apply it to the simplest possible eternal time machine solution in $\mathrm{AdS}_{3}$ based on two conical defects moving around their center of mass along a circular orbit. Closed timelike curves in this space-time extend all the way to the boundary of $\mathrm{AdS}_{3}$, violating causality of the boundary field theory. By use of the geodesic approximation we address the issue of self-consistent dynamics of the dual $1+1$ dimensional field theory when causality is violated, and calculate the two-point retarded Green function. It has a nontrivial analytical structure both at negative and positive times, providing us with an intuition on how an interacting quantum field could behave once causality is broken.
\end{abstract}

DOI: 10.1103/PhysRevD.94.044059

\section{INTRODUCTION}

Solutions to the equations of General Relativity that describe space-times containing closed timelike curves (CTC) have attracted significant interest as they revealed at least the hypothetical theoretical possibility of traveling in time. Since the renowned publication by Kurt Gödel [1] a number of causality violating solutions in GR as well as in modified theories of gravity have been constructed, among which we can name the Tipler-Van Stockum time machine generated by axially rotating distribution of particles [2,3], the Morris-Thorne-Yurtsever traversable wormhole [4,5], the Gott time machine based on moving conical defects [6], the Ori dust solution [7], and the solutions in $f(R)$ theories of gravity [8] and theories with nonminimal mattercurvature coupling [9].

All questions about physics of time machines that could be posed in principle fall into three general categories:

(i) Is there a physical way to create a time machine?

(ii) Is there any time machine solution that can be stable?

(iii) What dynamical behavior would a physical system experience evolving in a time machine background? None of the questions have yet received a definite answer.

The answer to the first question is believed to be negative. Extensive analysis of particular time machine solutions has demonstrated that in order to create a spacetime with CTC one needs matter that violates strong, weak or null energy conditions of General Relativity (different solutions require violation of different energy conditions), and only eternal time machines can exist $[10,11]$. However

\footnotetext{
*arefeva@mi.ras.ru †abagrov@science.ru.nl *saterskog@lorentz.leidenuniv.nl

${ }^{\S}$ kschalm@lorentz.leidenuniv.nl
}

we cannot be sure that all matter in the Universe obeys these conditions. For instance, there are a number of models of the dark energy violating the null energy condition [12-15], and this provides a way to by-pass the no-go statement.

The second question was raised by Hawking in [16], where he conjectured that a space-time with CTC can be stable only on the classical level, but will be unavoidably destroyed by quantum fluctuations of the metric. The real universal proof or refutal of the conjecture can be obtained only within a framework of a complete theory of quantum gravity. String theory opened a possibility to check the chronology protection condition in specific cases. In paper [17] devoted to the problem the authors have shown that the partition function of perturbative quantum strings in the ten-dimensional Gödel universe blows up due to the presence of closed timelike curves. These considerations were pushed forward in [18], where it was demonstrated that appearance of CTC in a certain (O-plane) orbifold background would cause a Hagedorn transition that restructures the space-time transforming it into a chronologically safe configuration. So these results can be considered as a very accurate and nice supporting evidence in favor of the Hawking conjecture. On the other hand in [19-22], it was demonstrated that the Gödel type solutions can be smoothly embedded in the context of string theory. Closed timelike curves in that case are hidden behind the so-called holographic screens and do not violate causality in the rest of the space-time. Thus the chronology is protected, but structure of the CTC remains unbroken by quantum effects. An approach to resolve CTC-caused pathologies by oxidizing supergravity solutions to higher dimensions was proposed in [23]. The relation between existence of naked closed timelike curves and nonunitarity of the microscopic description of the background was emphasized in [24]. 
An intriguing observation has been made by the authors of [25] and [26], that from the point of view of the AdS/CFT correspondence, the existence of CTC in the bulk can be related to negative or exceeding one fermionic probability in the boundary field theory. Per contra, in [27] it was demonstrated that, by deforming $\mathrm{AdS}_{5} \times S_{5}$ spacetime in a certain way, it is possible to construct a supergravity solution possessing CTC confined to the bulk, such that the stress-energy tensor of the dual boundary $\mathcal{N}=4$ superYang-Mills theory remains well defined and finite. Some issues related to holographic interpretation of CTC behind the horizon of the BTZ black hole were discussed in [28].

The third category includes various types of the "grandfather paradox." For a classical wave equation on a nonglobally hyperbolic space with CTC the possibility of self-consistent dynamics was demonstrated in [29,30]. Classical mechanical billiards and their self-consistency conditions have been studied in [31]. A basis of states of a free quantum field theory in the Gott time machine has been constructed in [32], where it was shown that the causality violation leads to an emergence of an effective nonunitary interaction in the theory. Nonunitarity of interacting field theories in time machines was analyzed in [33]. Some authors even argued that evolution of a physical system along closed timelike curves can be studied experimentally by mean of simulation of emergent gravity in metamaterials [34], or a qubit interacting with an older version of itself [35].

However the question about properties of an interacting quantum field theory in a time machine background remains open. When the notions of time ordering and unitarity are absent from the very beginning, it is unclear how to formulate an interacting field theory. In this paper we address this problem and by use of the AdS/CFT correspondence provide a constructive solution to it. Here we stand on the position that even if the presence of CTC causes breaking of unitarity in the boundary field theory [26], it should not be regarded as a big problem as long as we can formulate a prescription how to solve the theory. When one is trying to get an insight into physics of paradoxical systems, it is not very useful to rely on the "common sense" intuition and corresponding fundamental principles.

If the AdS/CFT correspondence is valid in the case of causality violation, it can provide an elegant possible way to address the paradox. In the large $N$ limit it relates quantum field theories to a classical gravity, and thus we can study properties of a quantum theory in the CTC background just by careful analysis of the dual Riemannian geometry, without any need to formulate special quantization rules that would only apply in the case of broken causality.

The paper is organized as follows. In the next section we introduce a simple setup for the time machine in $\mathrm{AdS}_{3}$. In Sec. III we discuss the geodesic structure of the spacetime, and suggest that it could lead to nontrivial effects in the boundary field theory. In Sec. IV we introduce a notion of timelike quasigeodesics that will be then used for connecting timelike separated boundary points. Finally, in Sec. V we formulate a precise algorithm for the Green function evaluation, provide the results of numerical simulations, and discuss the related phenomenology.

\section{TIME MACHINE IN $\mathbf{A d S}_{3}$}

The eternal time machine solution in AdS has been suggested by Gott and DeDeo in [36] (for similar solutions containing CTC but collapsing into a BTZ black hole see [37]). Here we briefly recall its structure closely following the original text.

The three-dimensional global anti-de Sitter space-time can be thought of as a hypersurface

$$
-X_{0}^{2}-X_{3}^{2}+X_{1}^{2}+X_{2}^{2}=-1,
$$

embedded in a four-dimensional flat $\mathbb{R}^{2,2}$ space-time with a metric:

$$
d s^{2}=-d X_{0}^{2}-d X_{3}^{2}+d X_{1}^{2}+d X_{2}^{2} .
$$

In the Schwarzschild coordinates the embedding formulas are

$$
\begin{aligned}
& X_{0}=\sqrt{1+R^{2}} \cos t, \\
& X_{3}=\sqrt{1+R^{2}} \sin t, \\
& X_{1}=R \cos \phi, \\
& X_{2}=R \sin \phi,
\end{aligned}
$$

where $R \in(0, \infty), t \in(-\infty, \infty), \phi \in[0,2 \pi)$.

The induced metric is then

$$
d s^{2}=-\left(1+R^{2}\right) d t^{2}+\frac{d R^{2}}{1+R^{2}}+R^{2} d \phi^{2} .
$$

A massive particle put into a three-dimensional space-time removes a wedge with an angle deficit proportional to the mass of the particle, and edges (faces) emerging from this pointlike particle. Points on the opposite edges of the wedge are identified, and the resulting space-time contains a conical defect, Fig. 1(a). When we are looking at the unfolding of the conical defect, coordinate locations of the edges do not have an independent physical meaning, and we are free to rotate them preserving the angular deficit. For our purposes it will be convenient to make the cut out "pizza slice" twist in time with a constant angular velocity in the reference frame of the massive particle, making a full rotation in a period $2 \pi$, Fig. 1 (b). Then for the trailing and leading faces of the wedge in the embedding coordinates we get 


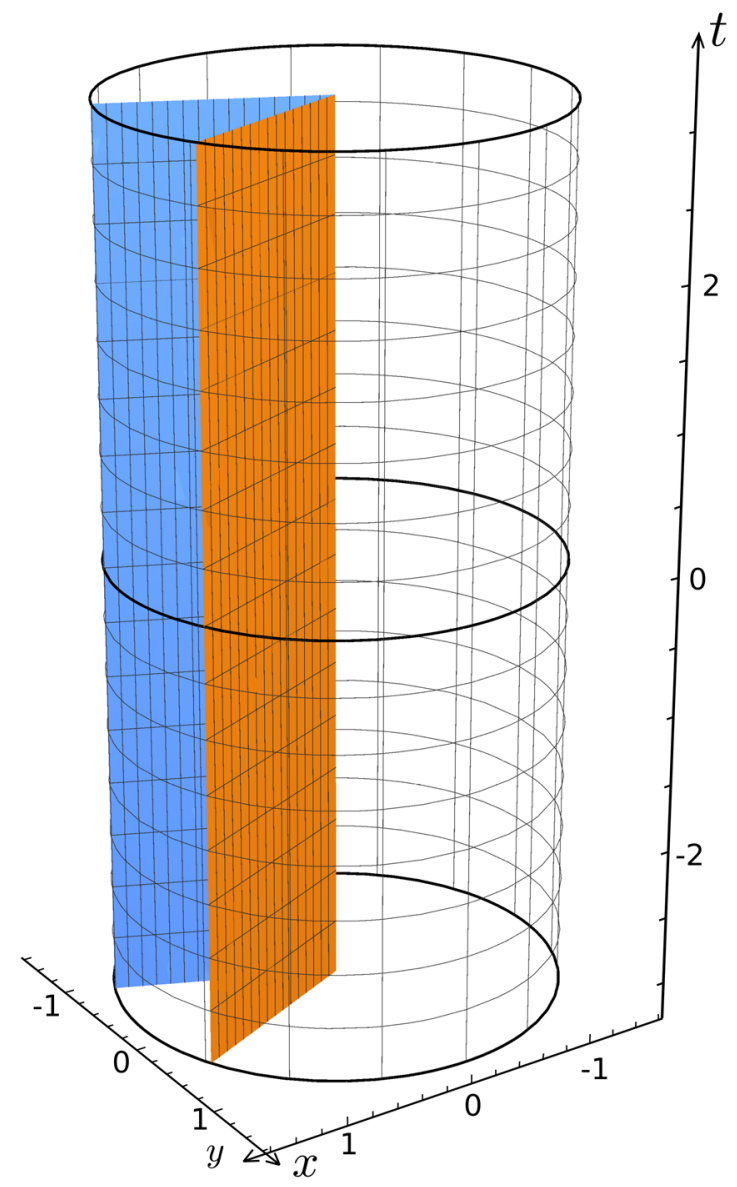

(a)

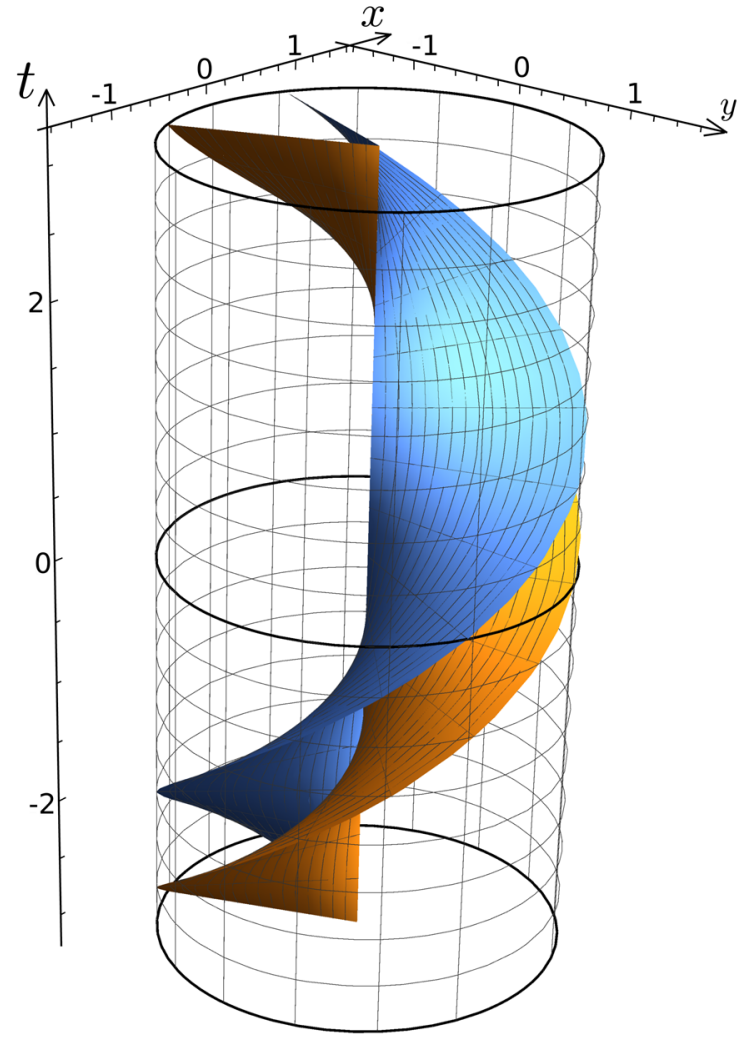

(b)

FIG. 1. Two equivalent unfoldings of the $\mathrm{AdS}_{3}$ spacetime with a conical defect $\alpha=\sqrt{3} \pi$. The larger part of the spacetime outside of the faces is to be cut out, and the faces are identified. The only physical space is the narrow region between the faces. To construct a time machine the twisted unfolding is more convenient to use.

$$
\begin{array}{ll}
X_{0}^{t}=\sqrt{1+R^{2}} \cos t & X_{0}^{l}=\sqrt{1+R^{2}} \cos t, \\
X_{3}^{t}=\sqrt{1+R^{2}} \sin t & X_{3}^{l}=\sqrt{1+R^{2}} \sin t, \\
X_{1}^{t}=R \cos (t-\alpha / 2) & X_{1}^{l}=R \cos (t+\alpha / 2), \\
X_{2}^{t}=R \sin (t-\alpha / 2) & X_{2}^{l}=R \sin (t+\alpha / 2) .
\end{array}
$$

Here $\alpha$ is the angular deficit of the conical spacetime. Integrating the spacetime stress-energy tensor over the angle, we can deduce that the effective mass concentrated in the interior of the bulk is

$$
M=-\frac{1}{8 G}+\frac{\alpha}{16 \pi G} .
$$

Here the second term is the mass of the pointlike source, and the first one is the contribution from the negative AdS curvature.

For a single static conical defect we cannot make its angular deficit $\alpha$ larger than $2 \pi$. If the mass of the pointlike particle exceeds the limit $\alpha=2 \pi(M \geq 0)$, the resulting space-time will be rather a BTZ black hole instead of a naked conical singularity [38].
However, if we boost the massive source, the removed wedge is effectively getting "squeezed" from the point of view of an external observer at rest [see Fig. 2(a)]. This provides a room for a second conical defect with a deficit angle $\beta$ such that $\alpha+\beta>2 \pi$. In other words, relative motion can support the system of conical defects, preventing them from collapsing into a black hole. This will be the essence of the DeDeo-Gott construction.

Consider a system of two identical conical defects undergone two opposite Lorentz transformations, in the $\left(X_{0}, X_{1}\right)$ and $\left(X_{3}, X_{2}\right)$ planes of the embedding space each:

$\Lambda_{I}=\Lambda_{I I}^{-1}=\left(\begin{array}{cccc}\cosh \psi & 0 & \sinh \psi & 0 \\ 0 & \cosh \psi & 0 & \sinh \psi \\ \sinh \psi & 0 & \cosh \psi & 0 \\ 0 & \sinh \psi & 0 & \cosh \psi\end{array}\right)$

In the three-dimensional coordinates of the AdS spacetime these Lorentzian transformations correspond to $S O(2,2)$ isometry transformations. 


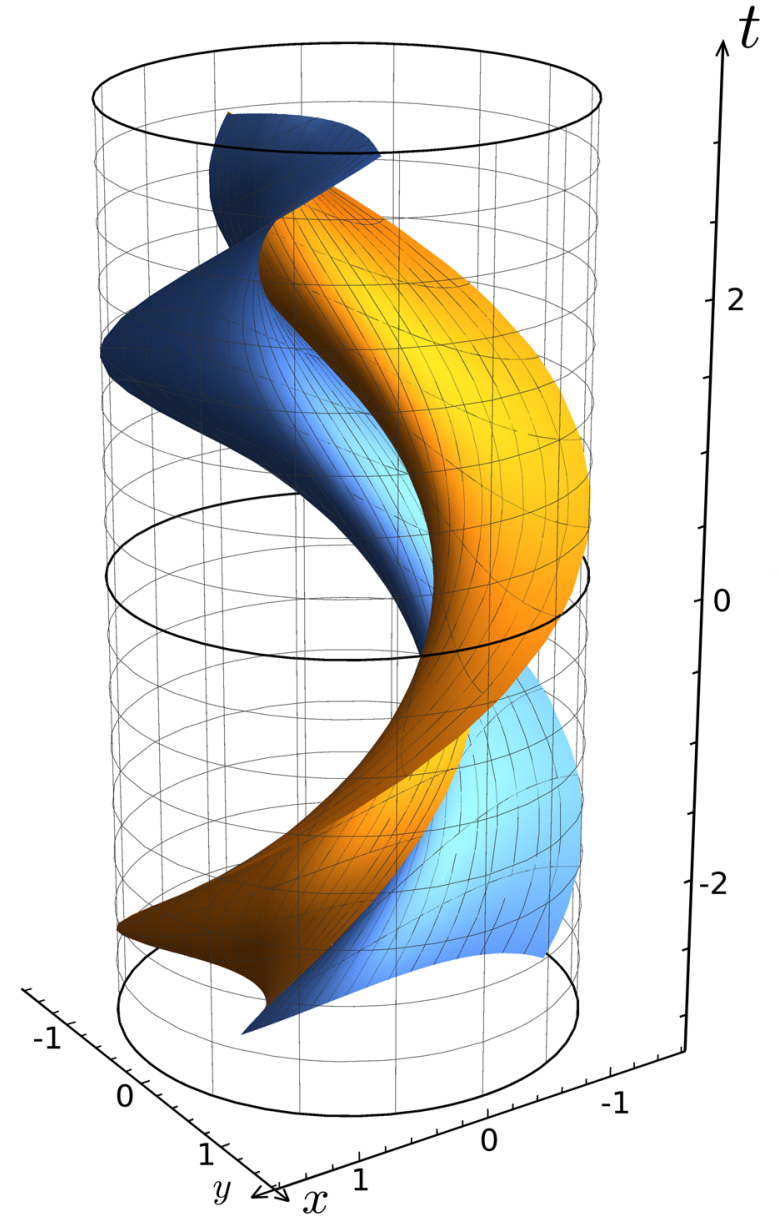

(a)

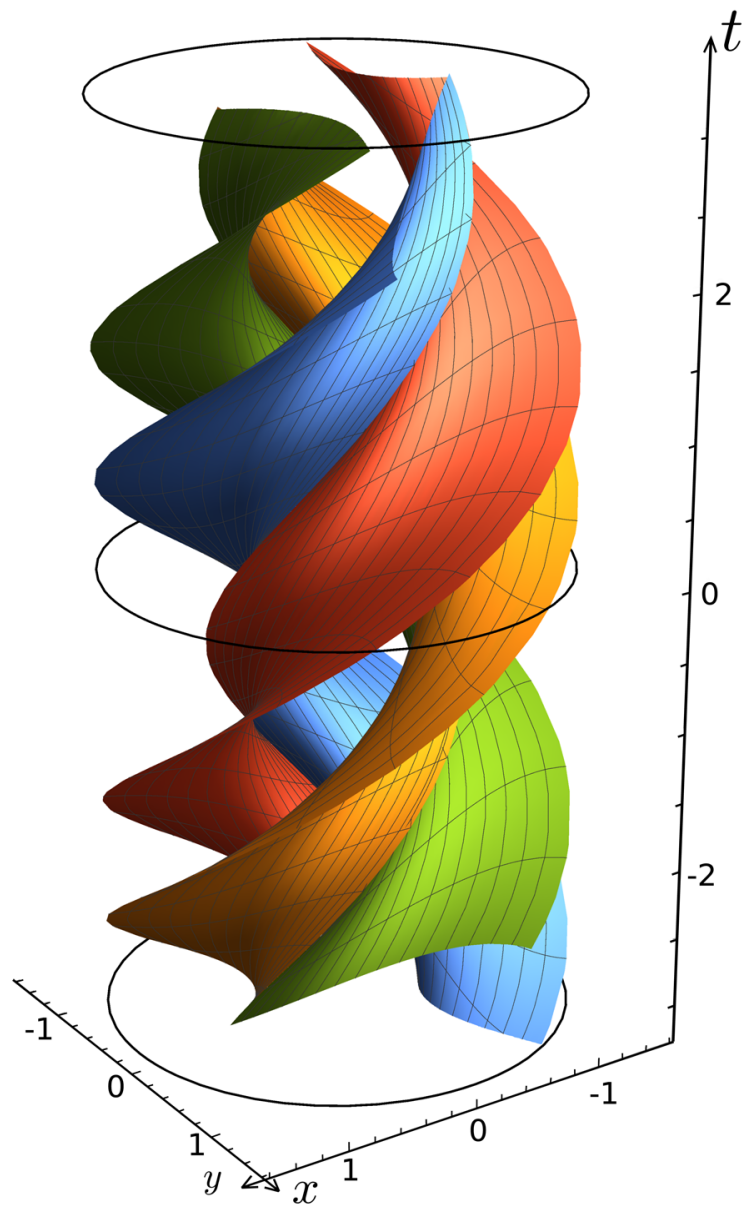

(b)

FIG. 2. (a) A boosted conical defect in $\mathrm{AdS}_{3}$. Faces of the wedge are deformed, and the identification occurs between points with different time coordinates (in the center-of-mass frame). Here $\alpha=\sqrt{3} \pi, \psi=1$. (b) The DeDeo-Gott time machine.

It can be shown that in the coordinates of global $\mathrm{AdS}_{3}$ these defects move along the same circular orbit $R=$ const with a constant velocity, always being at the opposite points of the orbit, Fig. 2(b). A conical defect sits at $\tilde{R}=0$ in its rest frame, i.e.

$\tilde{X}_{0}=\cos t, \quad \tilde{X}_{1}=0, \quad \tilde{X}_{2}=0, \quad \tilde{X}_{3}=\sin t$.

In the boosted frame

$$
\begin{aligned}
& X_{0}=\cosh \psi \cos t, \quad X_{1}=\sinh \psi \cos t, \\
& X_{2}=\sinh \psi \sin t, \quad X_{3}=\cosh \psi \sin t,
\end{aligned}
$$

hence

$$
\begin{gathered}
R=\sqrt{X_{1}^{2}+X_{2}^{2}}=|\sinh \psi|, \\
\cos \phi=\frac{X_{2}}{R}=\frac{\sinh \psi \cos t}{|\sinh \psi|}=\operatorname{sgn} \psi \cdot \cos t .
\end{gathered}
$$

In the rest frame of a wedge, the points on its edges are identified at equal coordinate times. However if we boost it, from the point of view of an external observer this identification would occur at different times leading to time jumps for a particle moving around the conical singularity. In the case of a single conical defect its boost can be regarded as a global coordinate transformation of the space-time, which obviously cannot cause any new physical effects. However, with two defects moving relative to each other, the relative time jumps become a physical effect that cannot be eliminated by a (proper) choice of coordinate system. These time jumps allow for the existence of CTC.

Existence of closed timelike curves in this space-time can be demonstrated by looking at the identification of the edges near the boundary of $\mathrm{AdS}_{3}$ (at $R \rightarrow \infty$ ). We refer the reader to [36] for a detailed discussion, here we just quote the result. Speaking in terms of the unfolding of the twoconical space-time, when a timelike particle living on the boundary of the AdS cylinder hits an edge of one of the two wedges, it undergoes a time and an angle jump: 


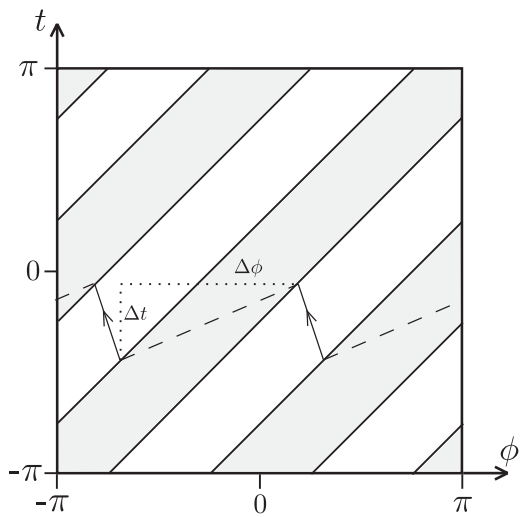

FIG. 3. A timelike particle moving along the boundary experiences a jump in time and angle when it hits a face of one of the wedges. If $\Delta t+\Delta \phi>\pi$, closed timelike curves become possible. The gray strips are the cut out part of the boundary, and the white strips are the physical part of the boundary.

$$
\begin{aligned}
& \Delta t=2 \arctan \left(\frac{\sin (\alpha / 2) \tanh \psi}{1+\cos (\alpha / 2) \tanh \psi}\right), \\
& \Delta \phi=2 \arctan \left(\frac{\sin (\alpha / 2)}{\tanh \psi+\cos (\alpha / 2)}\right) .
\end{aligned}
$$

It can be shown that $\Delta t+\Delta \phi=\alpha$. If $\alpha \geq \pi$, the world line of the particle becomes a closed timelike curve, and thus the space-time is a time machine, see Fig. 3.

In the regime when this limit is not exceeded, and CTC are not present, the space-time has been studied in detail from holographic point of view in [39], but the case of broken causality has not been addressed.

In the next sections we will study geodesic structure of this time machine and explicitly show that in the presence of the two orbiting conical defects we deal with a highly nontrivial lensing of geodesics, and this reflects on the structure of two-point Green's functions of the dual boundary theory. For holographic analysis of a single conical defect we refer the reader to $[40,41]$.

\section{WINDING OF GEODESICS AND CAUSALITY VIOLATIONS}

When conformal dimension $\Delta$ of a boundary operator in AdS/CFT is very high, the corresponding two-point Green function can be derived in the geodesic approximation [38]:

$$
G(A, B)=e^{-\Delta \mathcal{L}_{A B}},
$$

where $\mathcal{L}_{A B}$ is the length of a geodesic connecting boundary points $A$ and $B$. If there is more than one geodesic between $A$ and $B$, they can give additional contributions to the propagator. This is the case for the DeDeo-Gott time machine geometry, and here we address the possible outcome of this in detail.
Consider two arbitrary points $A\left(t_{1}, \phi_{1}\right)$ and $B\left(t_{2}, \phi_{2}\right)$ located in the physical (unremoved) part of the $\mathrm{AdS}_{3}$ boundary. Having two rotating conical defects in the bulk makes the structure of possible geodesics connecting ${ }^{1} A$ and $B$ very nontrivial, so we should find a way to calculate their contributions to the two-point Green's function $G(A, B)$. Let us shoot a geodesic from the boundary point $A$ to the point $B$. Before it hits the point $B$ it can undergo a number of "refractions" on the faces of wedges, winding around either of two conical defects clockwise (if it hits the leading face of the wedge head on) or counterclockwise (if it overtakes the trailing face of the wedge from behind). For example, schematically a typical geodesic may have a structure (see also Fig. 4)

$$
A \rightarrow \mathcal{W}_{I}^{-} \rightarrow \mathcal{W}_{I I}^{+} \rightarrow \mathcal{W}_{I}^{+} \rightarrow \mathcal{W}_{I I}^{+} \rightarrow B
$$

where $\mathcal{W}_{I, I I}^{+,-}$stands for the act of clockwise/ counterclockwise winding around the first or the second wedge respectively.

So, formally the Green's function in the geodesic approximation is given by

$$
G(A, B)=\sum_{n=0}^{\infty} \sum_{\left\{\mathcal{W}_{1} \cdots \mathcal{W}_{n}\right\}} e^{-\Delta \mathcal{L}\left(A\left\{\mathcal{W}_{1} \cdots \mathcal{W}_{n}\right\} B\right)},
$$

where the second sum is taken over all different winding structures corresponding to the same number of windings, and the first sum is taken over all winding numbers. ${ }^{2}$

It is easy to see that for a given number of windings $N$ the maximal possible number of topologically different geodesics is

$$
n_{N}=4 \cdot 3^{N-1}
$$

The first winding act can be of four different types. But for each of the next steps, if a geodesic wrapped around a conical defect, for example, clockwise, then on the next step it cannot go in the opposite direction and wrap around the same conical defect counterclockwise. It means that in the sequence of windings the winding act $\mathcal{W}_{I}^{+}$can be followed (at least hypothetically) by $\mathcal{W}_{I}^{+}, \mathcal{W}_{I I}^{+}, \mathcal{W}_{I I}^{-}$, but not by $\mathcal{W}_{I}^{-}$.

As we will see further, for a given pair of boundary points $(A, B)$ not all sequences of windings are physically realistic and can contribute to the sum (15).

\footnotetext{
${ }^{1}$ When $A$ and $B$ are timelike separated we encounter some subtleties caused by the fact that in the AdS space-time a timelike geodesic cannot reach the boundary. These issues will be commented further on, but the general point of view described in this section remains unchanged.

${ }^{2}$ This idea of windings, or entwinements, in holography has been introduced in [41], but there it was related to a concept of entanglement entropy "shadows" rather than to subleading contributions to the propagator.
} 


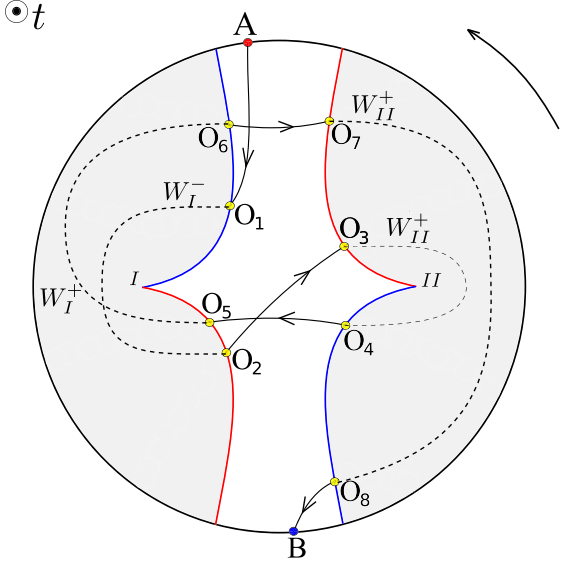

(a)

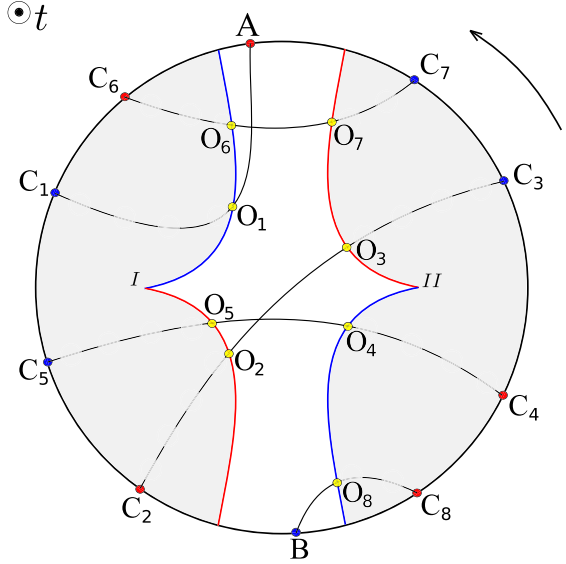

(b)

FIG. 4. Schematic constant time projection of a typical geodesic connecting points A and B in the time machine. Red curves are for leading faces of the rotating wedges, and blue curves are for trailing faces. The winding configuration in this particular case is $\mathcal{W}_{I}^{-} \mathcal{W}_{I I}^{+} \mathcal{W}_{I}^{+} \mathcal{W}_{I I}^{+}$according to the notations introduced in the main text. All shown points in principle can have different time coordinates (here we schematically project them down to a single time section, so the curves the geodesic is made from should be taken only as an approximate artistic representation). On picture (a) the acts of windings and identifications are shown explicitly. $A$ and $B$ belong to the physical unremoved part of the spacetime, and $O_{i}$ are the points where the geodesic undergoes "refraction" on the wedges. Picture (b) demonstrates the idea of complementary points $C_{i}$ located in the removed part of the spacetime.

To find a proper prescription for the lengths of the nontrivial winding geodesics let us discuss in detail a particular example.

Take a look at Fig. 4. The length of the presented geodesic is a sum of lengths of its composing arcs:

$$
\begin{aligned}
& \mathcal{L}\left(A \rightarrow \mathcal{W}_{I}^{-} \mathcal{W}_{I I}^{+} \mathcal{W}_{I}^{+} \mathcal{W}_{I I}^{+} \rightarrow B\right) \\
& \quad=\mathcal{L}_{A O_{1}}+\mathcal{L}_{O_{2} O_{3}}+\mathcal{L}_{O_{4} O_{5}}+\mathcal{L}_{O_{6} O_{7}}+\mathcal{L}_{O_{8} B} .
\end{aligned}
$$

We will refer to points $C_{i}$ as complementary points, and $O_{i}$ as refraction points. Coordinates of the refraction points $O_{i}$ are to be found from coordinates of $A$ and $B$, and that can be easily done step by step.

Focus on the first refraction on the wedge, $\mathcal{W}_{I}^{-}$. The points of interest are $\left\{A, O_{1}, C_{1}, C_{2}, O_{2}, C_{3}\right\}$. Here we neglect for a while the second wedge, so we do not consider the point $O_{3}$ at all, and we treat $C_{3}$ as a physical point (not just as a point in the complementary "removed" space), see Fig. 5(a). These six points can be regarded as a result of boost transformation $\Lambda_{I}$ applied to the wedge. We can "unboost" the wedge and find static preimages of these points [see Fig. 5(b)]. For the "unboosted" points the following relations trivially hold:

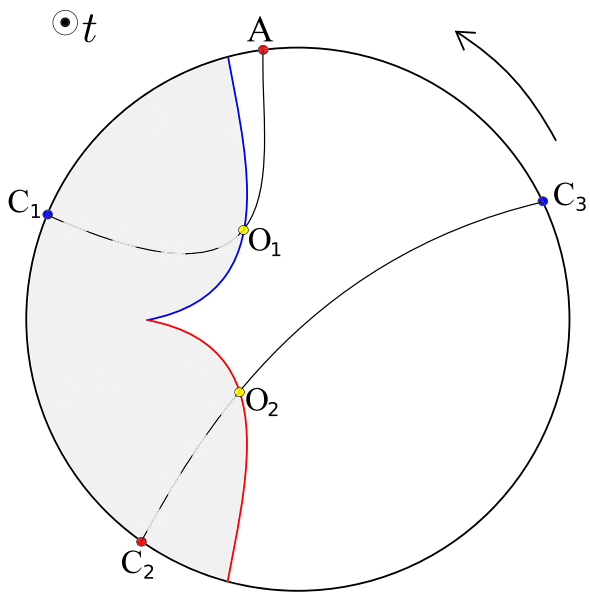

(a)

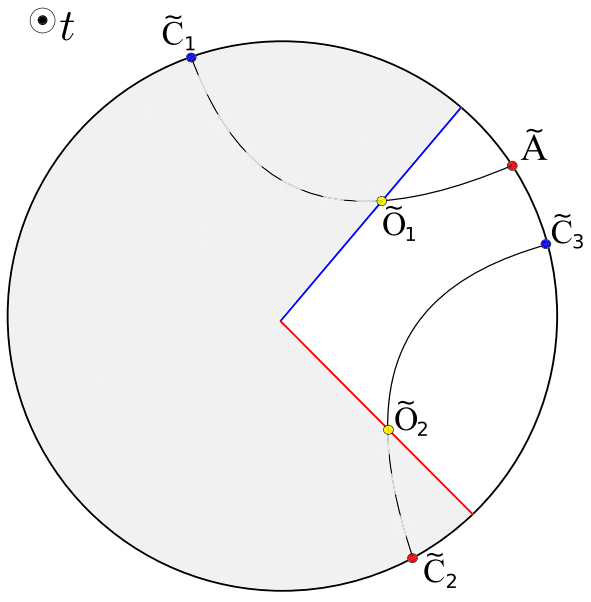

(b)

FIG. 5. The left picture demonstrates $\mathcal{W}_{I}^{-}$refraction of a geodesic on the first conical defect. The right picture demonstrates how it looks in a reference frame of the conical defect. Again, points generically belong to different time slices, and the representation is purely schematic. 


$$
\begin{aligned}
& \tilde{C}_{2}=\Lambda_{I}^{-1} C_{2}=\Lambda_{I}^{-1} A-(0, \alpha)=\tilde{A}-(0, \alpha), \\
& \tilde{C}_{3}=\Lambda_{I}^{-1} C_{3}=\Lambda_{I}^{-1} C_{1}-(0, \alpha)=\tilde{C}_{1}-(0, \alpha),
\end{aligned}
$$

where $(0, \alpha)$ is a boundary identification vector proportional to the angular deficit. Here we subtract the identification vector $(0, \alpha)$ because this particular winding is counterclockwise. For clockwise $\mathcal{W}^{+}$we should rather add $(0,+\alpha)$. In that case points $\tilde{O}_{1}$ and $\tilde{O}_{2}$ are intersections of geodesics $\tilde{A} \tilde{C}_{1}$ and $\tilde{C}_{2} \tilde{C}_{3}$ with faces of the static wedge.

Applying the same procedure to the other windings, in a generic case we get a system of recurrent relations

$$
\begin{aligned}
C_{2} & =\Lambda_{1}\left(\Lambda_{1}^{-1} A \pm(0, \alpha)\right), \\
C_{2 j} & =\Lambda_{j}\left(\Lambda_{j}^{-1} C_{2 j-2} \pm(0, \alpha)\right), \\
C_{2 N-1} & =\Lambda_{N}\left(\Lambda_{N}^{-1} B \mp(0, \alpha)\right), \\
C_{2 j-1} & =\Lambda_{j}\left(\Lambda_{j}^{-1} C_{2 j+1} \mp(0, \alpha)\right) .
\end{aligned}
$$

Here $\Lambda_{j}=\Lambda_{I}$ if the corresponding winding is $\mathcal{W}_{I}^{ \pm} . \Lambda_{j}=$ $\Lambda_{I I}$ if the corresponding winding is $\mathcal{W}_{I I}^{ \pm}$. In these formulas we pick up the upper sign if $\mathcal{W}_{I, I I}^{+}$, and the lower sign if $\mathcal{W}_{I, I I}^{-}$.

Note that the Lorentz boost we have defined in terms of the embedding space coordinates acts nonlinearly on the $\mathrm{AdS}_{3}$ points, therefore we cannot simply expand the parentheses in (19).

Then for each of the auxiliary $\operatorname{arcs} C_{2 k} C_{2 k+1}$ we can derive coordinates of the refraction points $O_{2 k}, O_{2 k+1}$, and write down corresponding lengths of the composing arcs.

Later we will also show that not every formally generated sequence of windings does exist.

\section{QUASIGEODESICS CONNECTING BOUNDARY POINTS}

To discuss causality properties of the dual boundary QFT, we will in particular need to consider boundary points with timelike separation. The conceptual problem we unavoidably encounter here is the absence of timelike geodesics connecting points on the conformal boundary of AdS. The equations for timelike geodesics can be derived from the following Lagrangian for a massive particle in AdS:

$$
-\left(1+R^{2}\right) \dot{t}^{2}+\frac{\dot{R}^{2}}{1+R^{2}}+R^{2} \dot{\phi}^{2}=-1
$$

Such a particle has two conserved momenta:

$$
\begin{gathered}
\mathcal{E}=\left(1+R^{2}\right) \dot{t}, \\
\mathcal{J}=R^{2} \dot{\phi} .
\end{gathered}
$$

Substituting them into the Lagrangian we obtain the radial equation of motion:

$$
\begin{gathered}
-\frac{\mathcal{E}^{2}}{1+R^{2}}+\frac{\dot{R}^{2}}{1+R^{2}}+\frac{\mathcal{J}^{2}}{R^{2}}=-1, \\
\dot{R}^{2}=-\left(1+R^{2}\right)\left(1+\frac{\mathcal{J}^{2}}{R^{2}}\right)+\mathcal{E}^{2} .
\end{gathered}
$$

Clearly the right-hand side of the equation turns negative as $R \rightarrow \infty$, and thus no real solution to this equation can exist.

In the case of a stationary spacetime the obstacle could be easily surmounted by performing the analytic continuation of the metric to the Euclidean signature, calculating the Green function in terms of Euclidean lengths of the geodesics, and making the inverse Wick rotation back to real time. However in our case we deal with a spacetime that is not only nonstationary, but which has no good global notion of time. Hence we are forced to stick to the Lorentzian time.

The way to implement the geodesic approximation for timelike separated boundary points in the single Poincaré patch has been suggested in [42]. Let us turn for a second to the single patch of the $\mathrm{AdS}_{3}$ spacetime, covered by the Poincaré coordinates:

$$
d s^{2}=-r^{2} d t^{2}+\frac{d r^{2}}{r^{2}}+r^{2} d x^{2} .
$$

Again, a massive bulk particle has two kinetic invariants:

$$
\begin{aligned}
& E=r^{2} \dot{t}, \\
& J=r^{2} \dot{x},
\end{aligned}
$$

but now we have two different classes of spacelike geodesics.

(i) For $J^{2}>E^{2}$ :

$$
\left\{\begin{array}{l}
r(\lambda)=\sqrt{J^{2}-E^{2}} \cosh \lambda \\
x(\lambda)=x_{0}+\frac{J}{J^{2}-E^{2}} \tanh \lambda \\
t(\lambda)=t_{0}+\frac{E}{J^{2}-E^{2}} \tanh \lambda
\end{array}\right.
$$

(ii) For $E^{2}>J^{2}$ :

$$
\left\{\begin{array}{l}
r(\lambda)=\sqrt{E^{2}-J^{2}} \sinh \lambda \\
x(\lambda)=x_{0}-\frac{J}{E^{2}-J^{2}} \operatorname{coth} \lambda . \\
t(\lambda)=t_{0}-\frac{E}{E^{2}-J^{2}} \operatorname{coth} \lambda
\end{array}\right.
$$

We will be interested in the geodesics of the second kind. As $\lambda=0$ these geodesics approach the point $r(0)=0$, which is the Poincare horizon of the half-AdS chart. Regarding the horizon as a single infinitely far point (as in the theory of complex functions), we can consider two 
disconnected spacelike geodesics possessing the same kinetic invariants $E$ and $J$, but emerging from two different timelike separated boundary points $A\left(t_{A}, x_{A}\right)$ and $B\left(t_{B}, x_{B}\right)$, as two branches of a single geodesic reaching the spatial infinity and returning back to the boundary. The length of such a geodesic will be divergent not only as $r \rightarrow \infty$ (the standard holographic UV divergence), but also as $r \rightarrow 0$, but this can be cured by an appropriate renormalization. ${ }^{3}$ The resulting expression for the renormalized length of the geodesic is simply

$$
\mathcal{L}=\ln \left(\left(t_{B}-t_{A}\right)^{2}-\left(x_{A}-x_{B}\right)^{2}\right),
$$

which gives the correct answer for the two-point correlation function of $(1+1)$-dimensional $\mathrm{CFT}^{4}$ :

$$
\begin{aligned}
G\left(t_{A}, x_{A} ; t_{B}, x_{B}\right) & =e^{-\Delta \mathcal{L}}=\frac{1}{\left(\left(t_{B}-t_{A}\right)^{2}-\left(x_{A}-x_{B}\right)^{2}\right)^{\Delta}}, \\
\Delta t^{2}>\Delta x^{2} &
\end{aligned}
$$

In the global AdS space-time the Poincaré horizon has no special physical meaning, but we can still try to generalize this procedure to this case.

The boundary field theory now is defined on $S^{1} \times \mathbb{R}^{1}$ spacetime, and the two-point Green function that we must be able to reproduce via the geodesic approximation has the form [43]

$$
G\left(t_{A}, \phi_{A} ; t_{B}, \phi_{B}\right)=\frac{1}{\left|\cos \left(t_{B}-t_{A}\right)-\cos \left(\phi_{B}-\phi_{A}\right)\right|^{\Delta}} .
$$

Note that this function is periodic both in angle and time. While the angular periodicity is obvious by construction, periodicity in time emerges because of the finite size effects: an excitation created at some point in space and time starts dissipating, but later recollects and revives due to the spatial periodicity.

We will need a function that defines angular separation between boundary points while properly maintaining the rotational invariance of the system. For instance, given two angular coordinates $\phi_{2}=\frac{7 \pi}{4}$ and $\phi_{1}=\frac{\pi}{4}$, the difference between them along the shorter arc is

\footnotetext{
${ }^{3}$ For details see Appendices B and D of [42].

${ }^{4}$ If $\Delta x^{2}>\Delta t^{2}$, the renormalized length is

$$
\mathcal{L}=\ln \left(-\left(t_{B}-t_{A}\right)^{2}+\left(x_{A}-x_{B}\right)^{2}\right),
$$
}

and the full Green function is

$$
G\left(t_{A}, x_{A} ; t_{B}, x_{B}\right)=\frac{1}{\left|\left(t_{B}-t_{A}\right)^{2}-\left(x_{A}-x_{B}\right)^{2}\right|^{\Delta}} .
$$

$$
D\left(\phi_{2}, \phi_{1}\right)=-\frac{\pi}{2} \neq \phi_{2}-\phi_{1} \text {. }
$$

Thus we should use

$$
D\left(\phi_{1}, \phi_{2}\right)=\bmod \left(\phi_{2}-\phi_{1}+\pi, 2 \pi\right)-\pi .
$$

Analogously, for the arithmetic average of two angular coordinates (that provides a point exactly at the middle of the shorter arc between $\phi_{1}$ and $\phi_{2}$ ),

$\Sigma\left(\phi_{1}, \phi_{2}\right)=\frac{1}{2}\left(\phi_{1}+\phi_{2}-2 \pi \theta\left(-\cos \left(\frac{1}{2}\left(\phi_{1}-\phi_{2}\right)\right)\right)\right)$,

where $\theta(x)$ is the Heaviside step function.

Note that the Green function (34) has a symmetry:

$$
G\left(t_{A}, \phi_{A} ; t_{B}, \phi_{B}\right)=G\left(t_{A}, \phi_{A} ; t_{B}+\pi, \phi_{B}+\pi\right),
$$

where points on the right-hand side can be spacelike separated while points on the left-hand side have timelike separation:

$$
\begin{aligned}
\left(t_{B}-t_{A}\right)^{2} & >D\left(\phi_{B}, \phi_{A}\right)^{2}, \\
\operatorname{but}\left(t_{B}-t_{A}+\pi\right)^{2} & <D\left(\phi_{B}+\pi, \phi_{A}\right)^{2} .
\end{aligned}
$$

This symmetry can be used to construct a disjointed spacelike geodesic, with two branches reattached at the Poincaré horizon, connecting timelike separated points.

If we represent the global $\mathrm{AdS}_{3}$ space-time as a cylinder, the Poincaré horizon consists of two planes cutting the cylinder at $45^{\circ}$. The orientation of the planes (as a rigid construction) can be chosen arbitrarily. Then consider a spacelike geodesic emerging from boundary point $A$ (see Fig. 6), and terminating at boundary point $B^{*}$. Somewhere in the bulk it has a turning point $P_{1}$ where its radial coordinate $R^{*}=R(0)$ is minimal.

Since we are free to choose the location of the Poincaré horizon, we can always orient it in such a way that the $A B^{*}$ geodesic intersects it at the turning point $P_{1}$. This point $P_{1}$ can be identified with a point $P_{2}=P_{1}+\left(\pi_{t}, \pi_{\phi}, 0_{R}\right)$, located on the other cutting plane. The arc $P_{1} B^{*}$ can be then rotationally translated to this point: $P_{1} \rightarrow P_{2}$, and then $B^{*} \rightarrow B$, where $B=B^{*}+(\pi, \pi)$. Hereafter we will use disjointed "quasigeodesics" of this $A P_{1} P_{2} B$ type to connect timelike separated points.

The explicit analytic expression for the quasigeodesics can be derived in the following way. Let us take the Poincaré chart geodesics (29), and rewrite them in coordinates of the embedding spacetime. The corresponding coordinate transformation is given by

\footnotetext{
${ }^{5}$ The fact that we have to use such timelike quasigeodesics makes our analysis different from the traditional studies of billiards on quotients of hyperbolic space [44].
} 


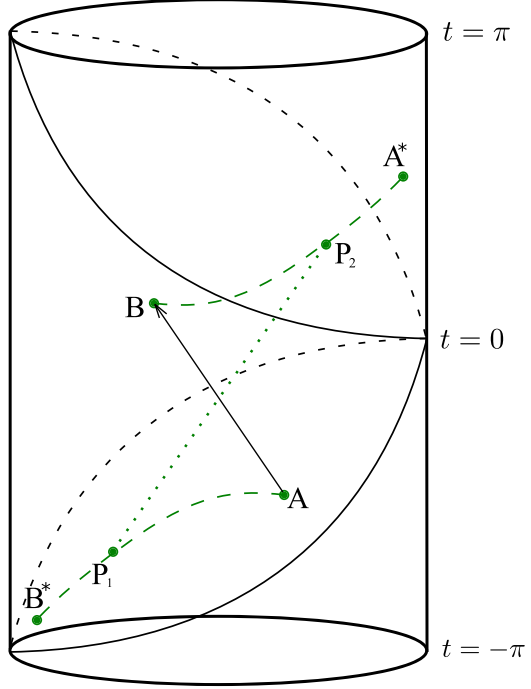

FIG. 6. A spacelike quasigeodesic connecting two boundary timelike separated points $A$ and $B$. A spacelike curve emerges from point $A$ and reaches the Poincaré horizon at point $P_{1}$. Then it jumps to a mirror point $P_{2}=P_{1}+\left(\pi_{t}, \pi_{\phi}, 0_{R}\right)$ on the second plane of the horizon, and proceeds further to $B$. The $\operatorname{arc} P_{2} B$ is a rotation of $P_{1} B^{*}$ by $\pi$.

$$
\begin{gathered}
X^{0}=\frac{r}{2}\left(\frac{1}{r^{2}}+1+x^{2}-t^{2}\right), \\
X^{1}=x r \\
X^{2}=\frac{r}{2}\left(\frac{1}{r^{2}}-1+x^{2}-t^{2}\right), \\
X^{3}=r t .
\end{gathered}
$$

Substituting here (29), and recalling the relations between the global and embedding coordinates (3), we obtain

$$
\begin{gathered}
\sqrt{1+R^{2}} \cos t=\frac{\left(-1+E^{2}-J^{2}\right)}{2 \sqrt{E^{2}-J^{2}}} \sinh \lambda, \\
\sqrt{1+R^{2}} \sin t=\frac{E}{\sqrt{E^{2}-J^{2}}} \cosh \lambda, \\
R \cos \phi=\frac{J}{\sqrt{E^{2}-J^{2}}} \cosh \lambda, \\
R \sin \phi=\frac{\left(-1-E^{2}+J^{2}\right)}{2 \sqrt{E^{2}-J^{2}}} \sinh \lambda .
\end{gathered}
$$

These can be solved to give us the embedding of the Poincaré chart spacelike geodesic into the global AdS:

$$
t(\lambda)=\arctan \left(\frac{2 E}{-1+E^{2}-J^{2}} \operatorname{coth} \lambda\right)+t_{0},
$$

$$
\begin{gathered}
\phi(\lambda)=\arctan \left(\frac{-1-E^{2}+J^{2}}{2 J} \tanh \lambda\right)+\phi_{0}, \\
R(\lambda)=\sqrt{\frac{J^{2}}{E^{2}-J^{2}} \cosh ^{2} \lambda+\frac{\left(-1-E^{2}+J^{2}\right)^{2}}{4\left(E^{2}-J^{2}\right)} \sinh ^{2} \lambda .}
\end{gathered}
$$

This quasigeodesic already has a $\pi$-jump in time at the turning point $\lambda=0$, and as explained before we also need to adjust the discontinuity in angle:

$$
\begin{aligned}
\tilde{\phi}(\lambda)= & \phi(\lambda)+\pi \theta(\lambda) \\
= & \arctan \left(\frac{-1-E^{2}+J^{2}}{2 J} \tanh \lambda\right) \\
& +\pi \theta(\lambda)+\phi_{0} .
\end{aligned}
$$

From now on we will omit the tilde.

The kinetic invariants can then be expressed in terms of the boundary coordinates:

$$
\begin{aligned}
& E=\frac{\sin \frac{t_{2}-t_{1}}{2}}{\sin \frac{D\left(\phi_{2}-\pi, \phi_{1}\right)}{2}-\cos \frac{t_{2}-t_{1}}{2}}, \\
& J=\frac{\cos \frac{D\left(\phi_{2}-\pi, \phi_{1}\right)}{2}}{\sin \frac{D\left(\phi_{2}-\pi, \phi_{1}\right)}{2}-\cos \frac{t_{2}-t_{1}}{2}} .
\end{aligned}
$$

The integration constants $t_{0}$ and $\phi_{0}$ in (49) can be represented as

$$
t_{0}=\frac{1}{2}\left(t_{1}+t_{2}\right), \quad \phi_{0}=\Sigma\left(\phi_{1}, \phi_{2}-\pi\right) .
$$

Inverting Eq. (50) we obtain dependence of the affine parameter on the radial coordinate:

$$
\lambda(R)= \pm \operatorname{arcsinh} \sqrt{\frac{4\left(E^{2}-J^{2}\right) R^{2}-4 J^{2}}{\left(-1-E^{2}+J^{2}\right)^{2}+4 J^{2}}},
$$

where the minus sign is taken on the first branch of the geodesic or quasigeodesic (i.e. before the turning point, when particle moves away from the boundary), and the plus sign is taken on the second branch (when particle moves towards the boundary). This function can be used to define the geodesic length, which is simply

$$
\mathcal{L}\left(R_{1}, R_{2}\right)=\lambda_{ \pm}\left(R_{2}\right)-\lambda_{ \pm}\left(R_{1}\right)
$$

for two points with radial coordinates $R_{1}$ and $R_{2}$.

Note that for a geodesic connecting two boundary points the length is divergent: 


$$
\begin{aligned}
\mathcal{L} & =\lim _{R \rightarrow \infty}\left(\lambda_{+}(R)-\lambda_{-}(R)\right) \\
& =2 \lim _{R \rightarrow \infty} \operatorname{arcsinh} \sqrt{\frac{4\left(E^{2}-J^{2}\right) R^{2}-4 J^{2}}{\left(-1-E^{2}+J^{2}\right)^{2}+4 J^{2}}}=\infty,
\end{aligned}
$$

and needs to be renormalized. The natural way to do it is to subtract the parameter independent divergent part, and define the geodesic length as

$$
\begin{aligned}
\mathcal{L}_{\text {ren }} & =\lim _{R \rightarrow \infty}\left(\lambda_{+}(R)-\lambda_{-}(R)-2 \ln R\right) \\
& =\ln \left(\frac{16\left(E^{2}-J^{2}\right)}{E^{4}-2 E^{2}\left(-1+J^{2}\right)+\left(1+J^{2}\right)^{2}}\right) .
\end{aligned}
$$

The argument of the logarithm is always positive for quasigeodesics connecting timelike separated points, but can be less than 1 . It means that the geodesic length after the renormalization in principle can be negative. Using (58) along with (52) we obtain the correct result for the retarded Green function:

$$
\begin{aligned}
G_{c}\left(t_{1}, \phi_{1} ; t_{2}, \phi_{2}\right)= & e^{-\Delta \mathcal{L}_{\text {ren }}} \\
= & \frac{1}{\left(\cos \left(t_{2}-t_{1}\right)-\cos \left(\phi_{2}-\phi_{1}\right)\right)^{\Delta}}, \\
& \text { where } \quad\left(t_{2}-t_{1}\right)^{2}>D\left(\phi_{1}, \phi_{2}\right)^{2},
\end{aligned}
$$

where $D\left(\phi_{1}, \phi_{2}\right)$ is the function introduced in (36). The possible negativity of the renormalized geodesic length is the reason why singularities of the correlator can be captured in the geodesic approximation.

Here we must pause for a second and stress Lorentz noninvariance of (59). The $S O(2,2)$ isometries of $\mathrm{AdS}_{3}$ are realized as the Lornetz boosts of the embedding $\mathbb{R}^{(2,2)}$ space (6). If we take two boundary points $A$ and $B$, and act on them with a bulk isometry transformation $\Lambda$ of this type, we will observe that it does not preserve the Green's function (59):

$$
G_{c}(\Lambda A, \Lambda B) \neq G_{c}(A, B) .
$$

This is caused by the fact that the bulk isometry transformations do not preserve the renormalized geodesic length (58) and the corresponding divergent counterterm separately. It is the fundamental difference between holography of a Poincaré chart and holography of global AdS. In the first case the cutoff can be fixed canonically in such a way that both finite and divergent parts of the geodesic are $S O(2,2)$ invariant, and as a result the boundary conformal transformations induced by the bulk isometries are Lorentzian boosts. So the Green function of a dual boundary field theory is a relativistic invariant object (33). In the second case the isometries rather act as generic conformal transformations leaving the Green function covariant, i.e. invariant up to some coordinate dependent scaling prefactors.

In the holographic language this is encoded in the fact that the renormalized lengths connecting boundary points are dependent on the choice of the reference frame. Below when we consider the DeDeo-Gott time machine geometry, we should be especially careful about this, since the geodesics there are combinations of Lorentz invariant and noninvariant terms as, for example, in (17). The proper way to deal with it is explained in the first subsection of Sec. V.

In the next section we will analyze lensing of the quasigeodesics on the conical defects and calculate the Green function of the dual field theory in the presence of the closed timelike curves in the bulk.

\section{THE TWO-POINT GREEN'S FUNCTION}

To make effects caused by the causality violation more explicit we will focus on the properties of the Green's function. We should be clear on what Green's function we shall compute. Naively the correct one is the retarded Green's function, but let us be more precise. In fact, the most clear question we can ask is whether locally causal propagation of a signal from the future to the past is possible. In order to discuss this issue we need to properly define what "retarded" means when chronology is not protected. This question seems to be very difficult to address in quantum theory in general, but here we can use the advantage of the AdS/CFT dictionary and define the property of retarded in bulk terms. Let us recall that evolution of a particle moving in the bulk of AdS can be split in two parts: "physical" continuous motion along a timelike or a spacelike (quasi)geodesic, and "topological" time jumps caused by winding around the conical defects. In the holographic language geodesic branches $C_{2 i} C_{2 i+1}$ (see Fig. 4) correspond to the continuous evolution, and $C_{2 i+1} \rightarrow C_{2 i+2}$ identifications correspond to the time jumps. In the absence of the closed timelike curves a signal could causally propagate from $A$ to $B$ if $B$ belongs to the future light cone of $A: B \succ A$. A natural generalization of this prescription for the time machine case is to impose that this should hold true for all physical segments, i.e. $C_{2 i+1} \succ C_{2 i} \forall i$. In other words, the value of the retarded Green's function evaluated between two boundary points is nonzero only if they can be connected by a quasigeodesic such that each of its segments connects causally separated points and is locally directed from the past to the future.

\section{A. The algorithm}

In Sec. III we have discussed the general idea of using the geodesic approximation to compute the boundary Green's function for the DeDeo-Gott geometry. Now we will formulate an exact algorithm for that. 
(i) Introduce a coordinate system on the unfolding of the double-cone space in such a way that the physical (unremoved) part of the boundary consists of two stripes covered by coordinate intervals:

$$
\begin{aligned}
t & \in(-\infty, \infty), \\
\phi & \in\left(-\frac{\pi}{2}-\frac{\Delta \phi}{2}+t,-\frac{\pi}{2}+\frac{\Delta \phi}{2}+t\right) \\
& \cup\left(\frac{\pi}{2}-\frac{\Delta \phi}{2}+t, \frac{\pi}{2}+\frac{\Delta \phi}{2}+t\right) .
\end{aligned}
$$

(ii) Fix two boundary points $A$ and $B$. For simplicity we can choose $A=\left(0,-\frac{\pi}{2}\right)$.

(iii) Fix the total number of windings $N$ that a geodesic of interest undergoes on the way from $A$ to $B$. In our simulations we will not go beyond $N=4$, because the higher-order contributions to the Green's function are highly suppressed.

(iv) For the given $A, B$, and the number $N$, generate all possible $4 \cdot 3^{N-1}$ sets of the complementary points $\left\{C_{1}, \ldots, C_{2 N}\right\}$ corresponding to different sequences of windings $\left\{\mathcal{W}_{1}, \ldots, \mathcal{W}_{N}\right\}$, using recurrent relations (19). The (quasi)geodesics then consist of $N+1$ arcs $A C_{1}, C_{2} C_{3}, \ldots, C_{2 N} B$, each of which is just a geodesic or a quasigeodesic ${ }^{6}$ curve in empty $\operatorname{AdS}_{3}$. An example of such a quasigeodesic for $N=2$ is shown in Fig. 7.

(v) Impose that each of the "odd" complementary points $C_{2 i+1}$ belongs to the causal future of the previous "even point": $C_{2 i+1} \succ C_{2 i}$. As discussed earlier, in this case they can be connected only with quasigeodesics.

(vi) For each of the causal quasigeodesics, solve for the intersection points $\left\{O_{1}, \ldots, O_{2 N}\right\}$. The easiest way to do this is to transform for each winding back to the rest frame of the corresponding wedge. For example, if branch $C_{2 i} C_{2 i+1}$ intersects first the trailing face of the second wedge, and then the leading face of the first wedge, we perform a Lorentz transformation of the branch to the second rest frame, then untwist the wedge by a simple coordinate transformation $\phi^{\prime}=\phi-t$, such that angular location of the face remains still in these corotating coordinates, and solve the equation

$$
\begin{gathered}
\left(\phi_{C_{2 i} C_{2 i+1}}^{I I}\left(\lambda_{2 i}\right)-t_{C_{2 i} C_{2 i+1}}^{I I}\left(\lambda_{2 i}\right)\right) \\
\bmod 2 \pi=\phi_{T_{I I}}^{\prime} \bmod 2 \pi .
\end{gathered}
$$

Then we repeat the procedure in the first rest frame:

$$
\begin{gathered}
\left(\phi_{C_{2 i} C_{2 i+1}}^{I}\left(\lambda_{2 i+1}\right)-t_{C_{2 i} C_{2 i+1}}^{I}\left(\lambda_{2 i+1}\right)\right) \\
\bmod 2 \pi=\phi_{L_{I}}^{\prime} \quad \bmod 2 \pi .
\end{gathered}
$$

\footnotetext{
${ }^{6}$ It depends on whether the points are spacelike or timelike separated.
}

(vii) Make sure that all these equations have real solutions (otherwise discard the geodesic).

(viii) Make sure that if a branch is not expected to intersect other faces within the physical region of the space, it actually does not (fake intersections within the removed part of the unfolding are allowed). In other words, if an arc $O_{2 i} O_{2 i+1}$ emerges from the face $L_{I}$ and terminates at the face $T_{I I}$, it should not have intersections with $L_{I I}$ and $T_{I}$.

(ix) Calculate the lengths of all inner segments of the geodesic $\left(\mathrm{O}_{2 i} \mathrm{O}_{2 i+1}\right)$. They are finite by construction and equal to

$$
L_{O_{2 i} O_{2 i+1}}=\lambda_{2 i+1}-\lambda_{2 i}
$$

(x) Renormalize the lengths of the boundary segments $A O_{1}$ and $O_{2 N} B$ as they are divergent:

$$
\begin{gathered}
L_{A O_{1}}=\frac{1}{2} L_{A C_{1}}^{\mathrm{ren}}+\lambda_{1}, \\
L_{O_{2 N} B}=\frac{1}{2} L_{C_{2 N} B}^{\mathrm{ren}}-\lambda_{2 N} .
\end{gathered}
$$

(xi) Calculate the renormalized lengths of $L_{A O_{1}}$ and $L_{O_{2 N} B}$ in the original frame. As mentioned in the previous section, the renormalized lengths are not Lorentz invariant. So, while we are free to constantly switch between different reference frames in order to calculate lengths of the finite inner segments $O_{2 i} O_{2 i+1}$, the renormalized lengths of the two boundary segments must be calculated in the original frame where we define the Green's function. In our case it is the "center-of-mass frame," where the two conical defects are symmetrically boosted.

(xii) Finally calculate contribution of the geodesics to the Green function:

$$
G(A, B)=\sum_{k} e^{-\Delta L_{k}}
$$

where the index $k$ runs over the set of geodesics that satisfy aforementioned conditions.

In accordance with the described algorithm we subsequently account for contributions from higher winding numbers starting with $N=2 .^{7}$ In other words, we formulate a kind of "perturbation theory" with the number of windings as a control parameter.

One property of this series expansion must be commented on. Each geodesic contributes to the Green function exponentially:

\footnotetext{
${ }^{7}$ For negative times $N=1$ windings do not contribute as they are due to the lensing on a single conical defect, that obviously cannot lead to time traveling. But for positive times we take them into account.
} 

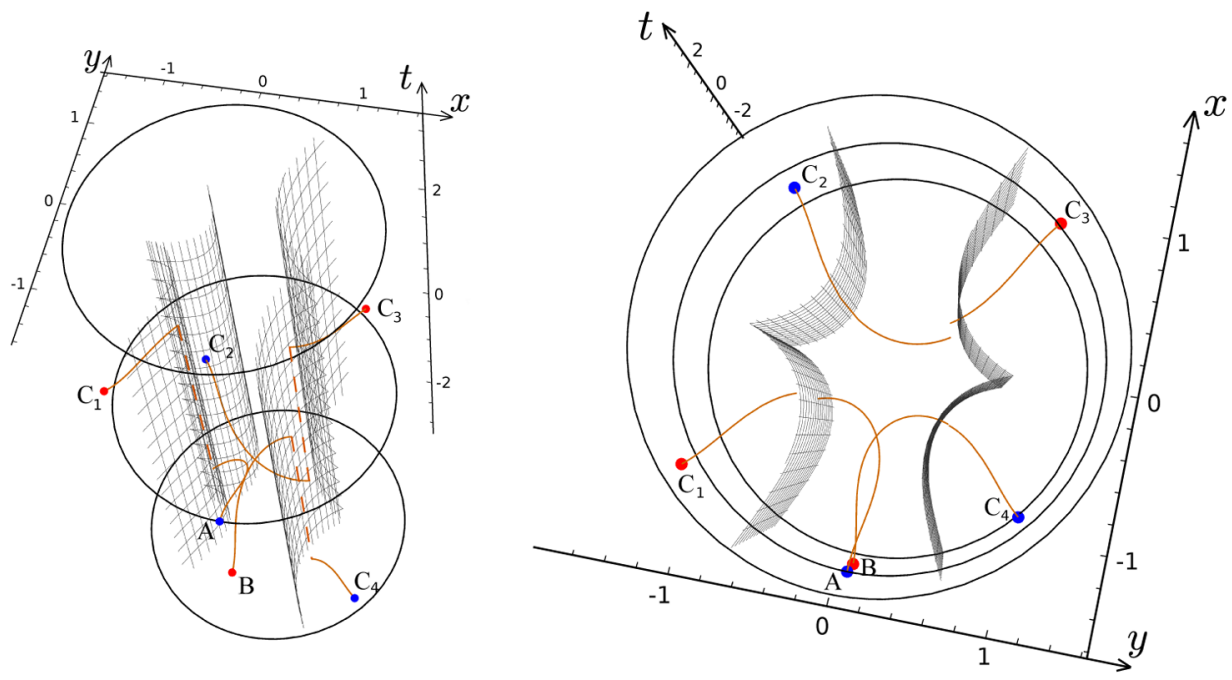

FIG. 7. A quasigeodesic with winding number $N=2$ connecting boundary points $A$ and $B$.

$$
e^{-\Delta L_{\text {ren }}}
$$

For higher windings the number of internal segments $C_{2 i} C_{2 i+1}$ of the geodesic grows linearly in $N$, and so does its renormalized length $L_{\text {ren }}$. Therefore the corresponding contribution to the Green function is exponentially small in a generic case when $L_{r e n}>0$. On the other hand the total number of possible topologically different geodesics scales as

$$
n \sim 3^{N-1},
$$

i.e. grows exponentially. Therefore in principle these two effects can compete and we cannot say a priori that the higher order contributions to the Green function are suppressed, and the sum over windings is convergent. If not, this could mean that our setup is unstable and undergoes a Hagedorn-like transition.

However there are three different reasons for it not to happen. First, by no means all of these $4 \cdot 3^{N-1}$ winding configurations satisfy the causality condition: $C_{2 i+1}>C_{2 i}$. Second, even if the causality condition for the set of complementary points is satisfied, the geometric structure of the geodesics becomes more and more complicated as the number of windings increases, and it becomes hard to force a geodesic curve to undergo the concrete sequence of windings [it is easy to see in Fig. 8(b)]. ${ }^{8}$ Finally, the "decaying" exponent has a conformal dimension as a knob, so at large enough $\Delta$ it dominates over the "growing" exponent, and the resulting Green's function can be made finite.

In the subsequent sections we will provide details of concrete calculations, but already these arguments

\footnotetext{
${ }^{8}$ The computational cost of generating the geodesics is unfortunately too high to check this numerically beyond $N=4$ order.
}

demonstrate that the dual boundary system appears to remain computationally under control. When the field theory enters the regime of causality violation, its Green's function remains well defined. This does not yet provide a true answer to the grandfather paradox or a proof that noncausal dynamics is in some way consistent. For that we need to go beyond the geodesic approximation and check "unitarity" (Huygens' principle $G(A, B)=\int G(A, C) G(C, B) d C$ ). Nevertheless, it indicates that AdS/CFT might supply a controlled computational scheme.

\section{B. Phenomenology and discussion}

We are now ready to implement our computational algorithm for the DeDeo-Gott geometry. As shown in Sec. II, closed timelike curves in the spacetime are present when the total angular deficit is more than $2 \pi$. For concreteness we impose $\alpha_{I, I I}=\sqrt{3} \pi$, and the boost rapidities $\psi_{I, I I}= \pm 1$. In their corresponding rest frames (in the corotating coordinates) the locations of the edges are taken to be

$$
\begin{aligned}
& \phi_{L_{1}}^{\prime}=\alpha / 2 \quad \phi_{T_{1}}^{\prime}=-\alpha / 2, \\
& \phi_{L_{2}}^{\prime}=\alpha / 2+\pi \quad \phi_{T_{2}}^{\prime}=-\alpha / 2+\pi .
\end{aligned}
$$

It is more convenient to calculate the Green function also in the corotating coordinates:

$$
G^{\mathrm{cr}}\left(t_{1}, \phi_{1}^{\prime} ; t_{2}, \phi_{2}^{\prime}\right)=G\left(t_{1}, \phi_{1}-t_{1} ; t_{2}, \phi_{2}-t_{2}\right) .
$$

For simplicity we will mostly study the Green function on a one-dimensional timelike line passing through point $A$, Fig. 9. We should be careful here. Any timelike line originated in one physical strip crosses the cut out region and enters the second strip. To formulate a quantum field theory on both strips simultaneously is possible yet tricky 


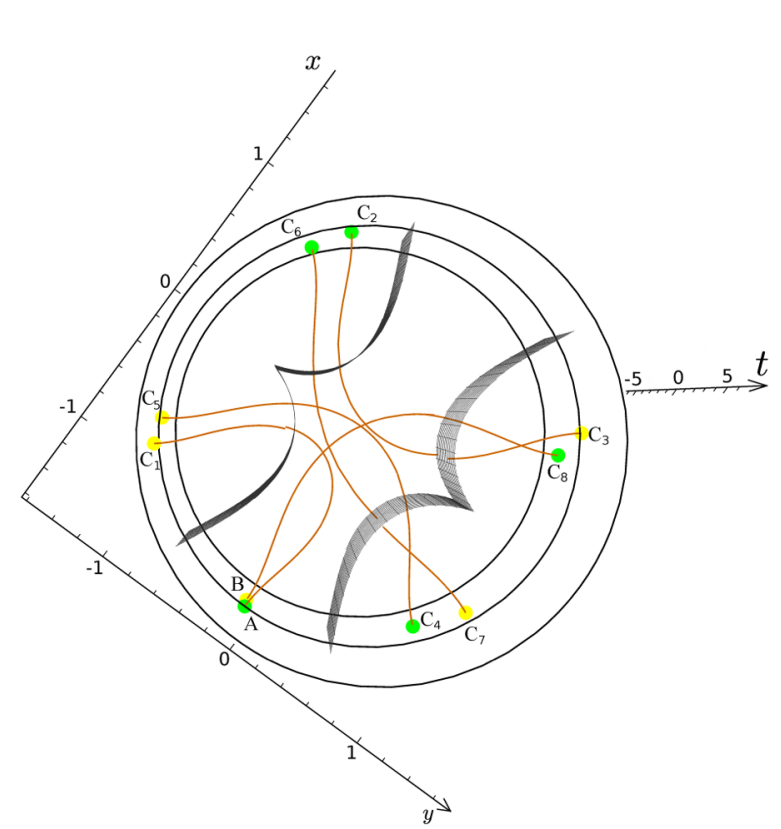

(a)

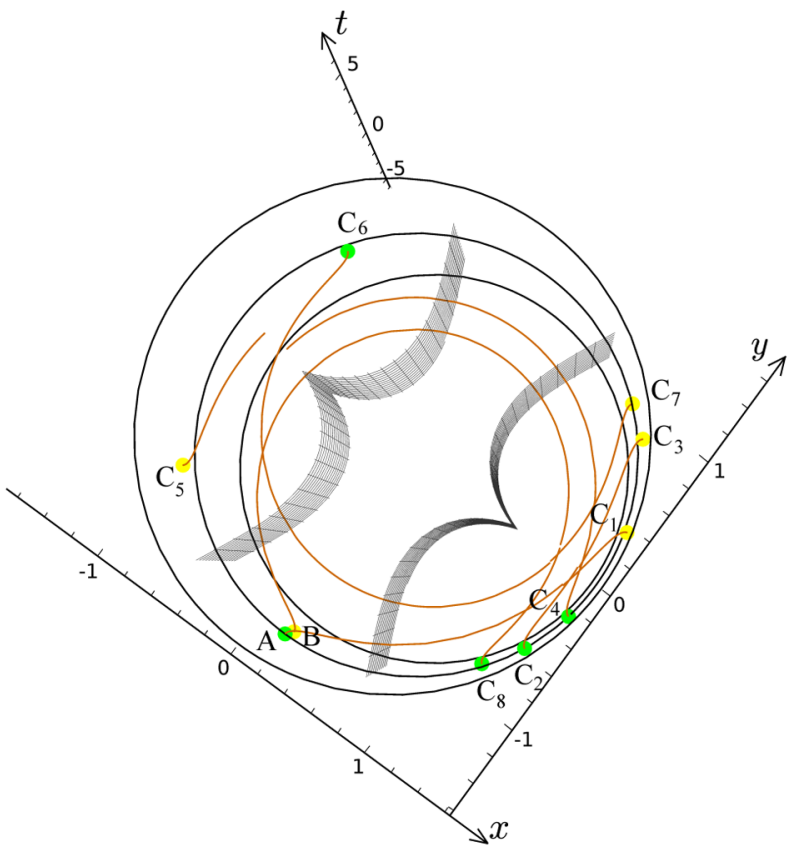

(b)

FIG. 8. (a) An example of a physical $N=4$ geodesic contributing to the Green function $\left(\mathcal{W}_{I}^{+} \mathcal{W}_{I I}^{+} \mathcal{W}_{I}^{+} \mathcal{W}_{I I}^{-}\right)$. (b) An example of physically impossible winding: for a given sequence of windings $\left(\mathcal{W}_{I I}^{+} \mathcal{W}_{I I}^{+} \mathcal{W}_{I}^{+} \mathcal{W}_{I I}^{+}\right)$a geodesic cannot be fit in the unremoved part of the spacetime. Quasigeodesic segment $C_{2} C_{3}$ lies completely in the removed unphysical part of the spacetime, and the other segments intersect the faces of the wedges in a wrong way (for instance, $C_{8} B$ should intersect only the trailing face of the second wedge, $T_{I I}$, instead it intersects $L_{I}, L_{I I}, T_{I}$ ).

due to the fact that on the unification of two parts of the boundary time cannot be globally defined. To avoid this difficulty we will consider the Green function on a timelike line in a close vicinity of the generatrix of the light cone. Then in a large range of times we will stay within one strip of the boundary.

In other words, the object we will attempt to evaluate is (in the corotating frame)

$$
G^{\mathrm{cr}}(0,-\pi / 2 ; t,-\pi / 2+\epsilon t), \quad \epsilon \ll 1 .
$$

We have performed the numerical calculation of the retarded Green function for negative times $t<0$ up to $N=4$ order, and for positive times $t>0$ up to $N=2$.

Let us discuss first the analytic behavior of the Green function at negative times, how the quantum particle behaves traveling back in time. A naive expectation would be to think that the Green function decays as $t \rightarrow-\infty$, and it is partially true. However as we can see at not very large negative times the function develops a number of nontrivial features, peaks which we can interpret as the "most probable" regions of times the particle can reach using the time machine. The corresponding results are present in Fig. 10. As an illustration we also provide a twodimensional plot for the leading $N=2$ winding at negative times, Fig. 11.

The origin of these peaks can be traced back to the fact that the renormalized length of a geodesic can be negative.
Generically at small conformal weights $N=2, N=3$ and $N=4$ contributions are commensurate, but already at $\Delta \gtrsim 2$, higher winding terms are suppressed as compared to $N=2$. However at specific points, where $\mathcal{L}_{\text {ren }}<0$, the corresponding contributions to the Green function are

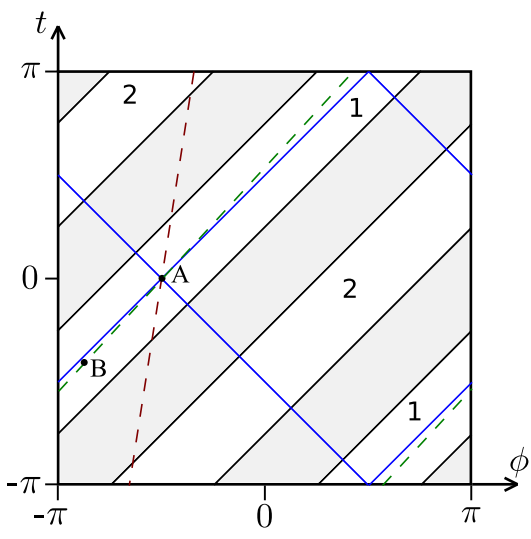

FIG. 9. The Green function is sourced at point $A$ with coordinates $(0,-\pi / 2)$. Blue lines depict the light cone emerging from this point. Any timelike line would cross the removed region and enter another strip (red dashed line). In order to avoid formulating the boundary field theory on both strips simultaneously we calculate the Green function on a timelike line very close to the light cone generatrix. 


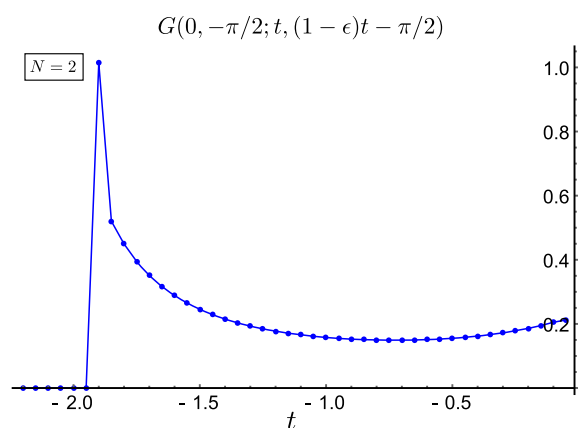

(a)

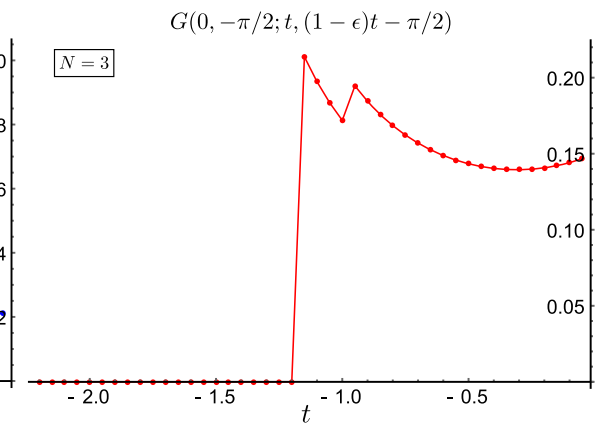

(b)

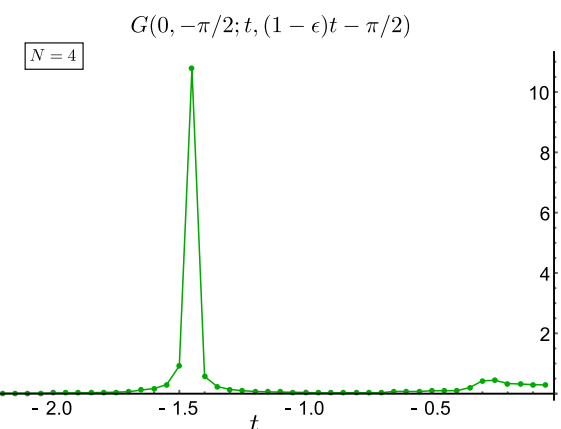

(c)

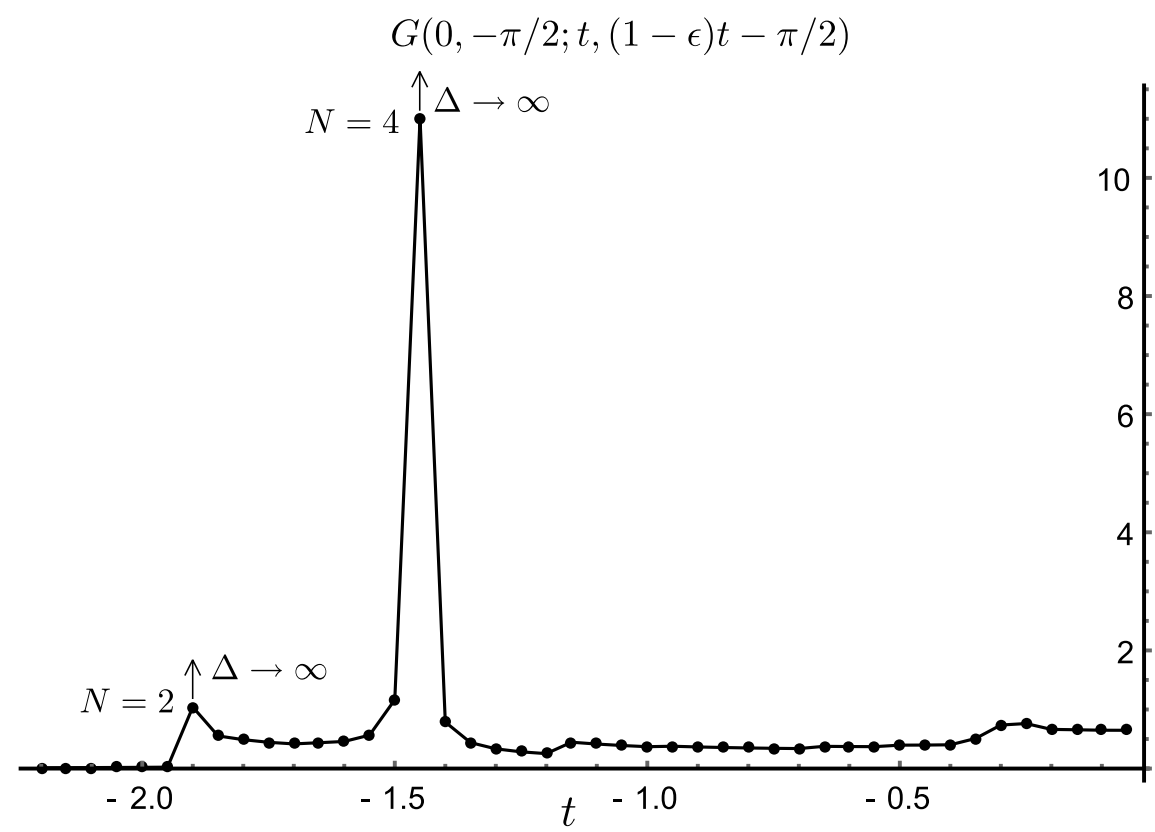

(d)

FIG. 10. (a) $N=2$, (b) $N=3$ and (c) $N=4$ contributions to the retarded Green function at negative times at $\Delta=1.5$. Discontinuities of the curves are artifacts of the geodesic approximation. (d) The retarded Green function at $\Delta=1.5(N=2, N=3$ and $N=4$ contributions are added up). For the large conformal dimensions peaks are enhanced, not suppressed, and we can see revival of the particle at moments preceding the excitation of the Green function. A not very large conformal dimension is chosen for convenience of presentation. Here $\epsilon=0.1$.

getting enhanced in the large $\Delta$ limit, $^{9}$ forming a sharp peak. For instance, $N=2$ set of geodesics contains such a curve around $t_{2}=-1.9$, and $N=4$ set has a special point at $t_{2} \simeq-1.45$, Fig. 10 .

We have not performed numerical simulations for $N>4$, but we cannot exclude that such negative length curves can appear also at large $N$. The geodesic length is defined by lengths of internal segments (always positive) and lengths of the two boundary segments (that in principle can be negative):

$$
\mathcal{L}_{A B}^{\mathrm{ren}}=\mathcal{L}_{A O_{1}}^{\mathrm{ren}}+\mathcal{L}_{O_{2 N} B}^{\mathrm{ren}}+\sum_{i=1}^{N} \mathcal{L}_{O_{2 i-2} O_{2 i-1}} .
$$

\footnotetext{
${ }^{9}$ Strictly speaking, the geodesic approximation is reliable only in this limit.
}

If $\mathcal{L}_{A O_{1}}^{\text {ren }}+\mathcal{L}_{O_{2 N} B}^{\text {ren }}<0$, and $\left|\mathcal{L}_{A O_{1}}^{\text {ren }}+\mathcal{L}_{O_{2 N} B}^{\text {ren }}\right|>\sum_{i=1}^{N} \mathcal{L}_{O_{2 i-2} O_{2 i-1}}$, the Green function will get a contribution that does not vanish in the large $\Delta$ limit. For a large number of internal segments it is not likely, but neither is impossible: while all internal lengths are finite, the renormalized negative lengths might be of an arbitrarily huge absolute value:

$$
L_{A O_{1}}^{\text {ren }}<0, \quad\left|L_{A O_{1}}^{\text {ren }}\right| \gg 1,
$$

thus dominating over positive contributions.

In the case of a large conformal dimension it would mean that, if we were able to sum up contributions in all winding orders, the resulting Green's function would have a shape of a comb with a number of peaks (in our calculations we discovered two of them). These peaks play a role of "pit stops" for a particle traveling in time; they form a set of 


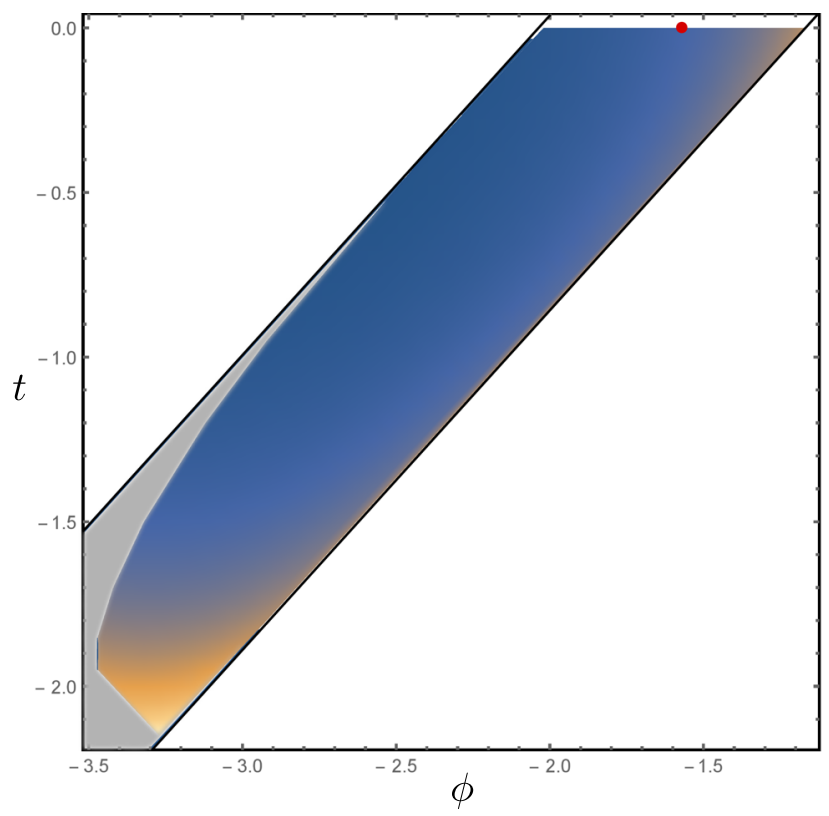

FIG. 11. Density plot of $N=2$ contribution to the retarded Green function at $\Delta=1.5$ at negative times in two dimensions. The Green function is sourced at the red point $(0,-\pi / 2)$. The deep blue region corresponds to low values of $G_{R}$, while the yellow region corresponds to its peak. We construct the theory only within one of the two strips of the boundary. The sudden break of the function signals that some regions of the spacetime are unattainable for the $N=2$ quasigeodesics.

easily reachable coordinates in time. Hence we deal with specific "negative time" revivals.

On its own, these revivals as due to nontrivial winding of geodesics are not specific to the existence of CTC $[45,46]$.

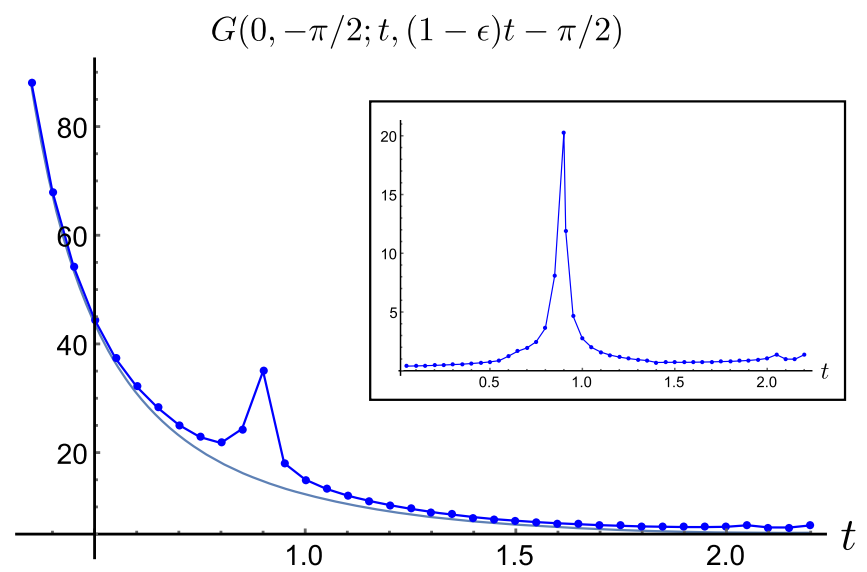

FIG. 12. The retarded Green function at conformal weight $\Delta=$ 1 at positive times. The plot demonstrates how the nontrivial $N=2$ windings modify the original Green function $(N=1$ does not contribute when we consider the boundary theory within a single strip). The offset plot represents the $N=2$ winding contribution separately. We have made the Green's function timelike to avoid dealing with the light-cone singularity. Here $\epsilon=0.1$
They are also present at positive times (Fig. 12). It is quite remarkable, however, that negative time revivals also exist. These are clearly correlated with the existence of CTC, and the existence of classical geodesic trajectories that take one "back in time." Do note that they are a special feature. Even in the absence of negative time revivals the effects of CTC still appear in the form of nonvanishing weight at negative times. If $\alpha<\pi$, and CTC are not present in the bulk, the retarded Green's function is identically zero within the past light cone.

\section{PHASES OF THE BOUNDARY FIELD THEORY}

We have calculated the Green's function numerically up to $N=4$ windings for the time machine geometry with $\alpha=\sqrt{3} \pi$ and $\psi=1$. However it would be interesting to study how the properties of the Green function change upon changing the strength and rapidities of the conical defects. We constructed the leading order $N=2$ contribution to

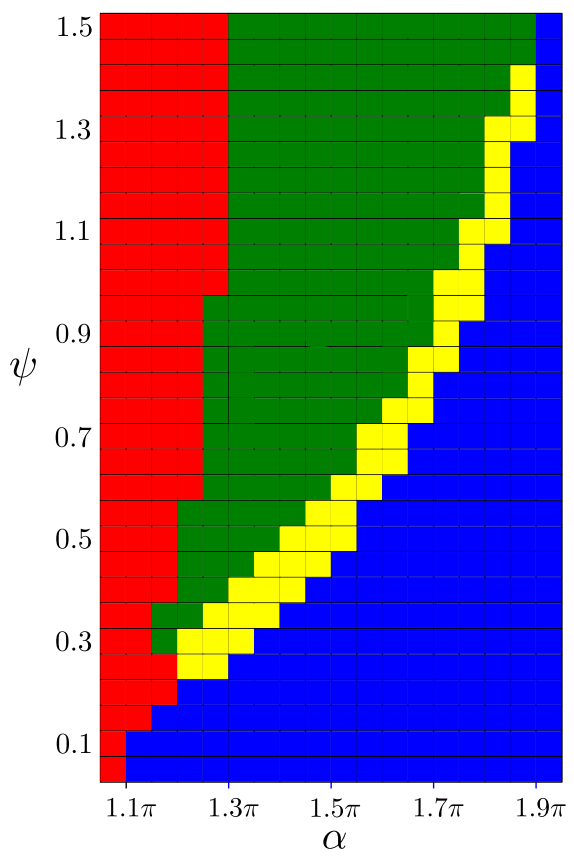

FIG. 13. The four different "phases" of the boundary field theory. Blue spots depict the region of forbidden geometries, yellow spots are for the phase of negative time revivals, green spots form the region where the retarded Green function is nonzero at negative times, but does not exhibit reviving peaks in the large $\Delta$ limit. Red spots are where at the leading $N=2$ order the boundary field theory retarded Green function does not demonstrate causality violation [i.e. $G^{\mathrm{cr}}(0,-\pi / 2 ; t,-\pi / 2) \equiv 0, t<0$ ] despite the presence of the CTC in the bulk. Everything is based on the numerical simulations of the leading $N=2$ contribution to the retarded Green function. We expect higher order corrections to change the diagram qualitatively, but not quantitatively. 


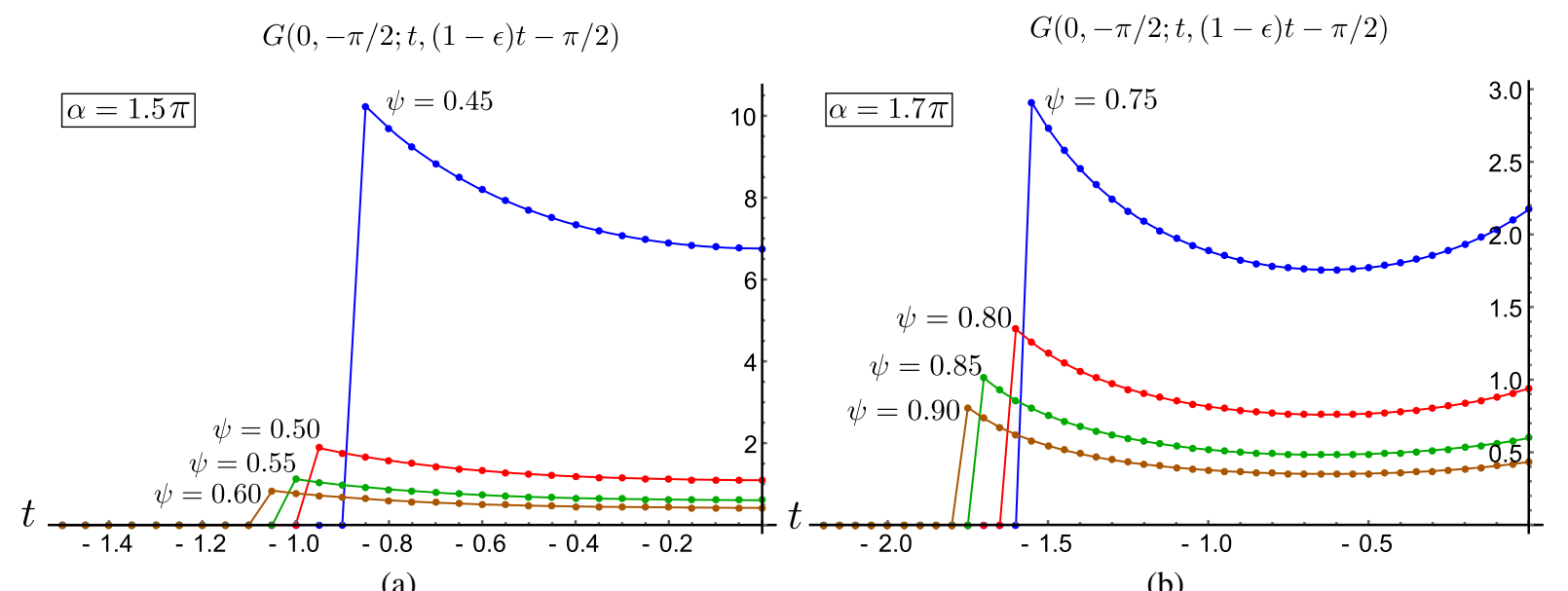

(a)

(b)

FIG. 14. The negative time $N=2$ contribution to the retarded Green's function at (a) $\alpha=1.5, \psi=0.45,0.50,0.55,0.60$, and (b) $\alpha=1.7, \psi=0.75,0.80,0.85,0.90$. Here $\Delta=1$. On each of the plots the first three peaks are getting stronger and sharper as $\Delta \rightarrow \infty$ (the yellow region on the phase diagram), while the fourth one is getting suppressed in the same limit (the green region), so we do not consider it to be an actual revival of a noncausal excitation. Counterintuitively, the weight of the Green function drastically decreases when we increase $\alpha$ or/and $\psi$.

the retarded Green function at negative times for $\alpha \in$ $(1.1 \pi, \ldots, 1.95 \pi)$, and $\psi \in(0.1, \ldots, 1.5)$ with stepping $\Delta \alpha=0.05 \pi, \Delta \psi=0.05$, paying special attention to the location in time and strength of the revival peak.

The results can be schematically summarized in the form of a phase diagram, Fig. 13:

(i) If for a given value of $\alpha$ the rapidity $\psi$ is not large enough to prevent the system from collapsing, the DeDeo-Gott geometry is forbidden (blue).

(ii) If for a given value of $\alpha$ the rapidity $\psi$ allows for the existence of the DeDeo-Gott time machine, but still not very large, we clearly see the effect of revival, and the peak is sharper the closer $\psi$ is to the lower bound (yellow).

(iii) If the rapidity is too large, causality is violated, but excitations just decay and do not revive at negative times anymore (green).

(iv) At very small values of $\alpha$ the retarded Green function does not exhibit any nontrivial features at negative time even in the presence of the closed timelike curves (red). However, this feature is likely just an artifact of $N=2$ approximation, and we do not expect it to be there for higher windings.

The profiles of the Green's function at negative times are presented in Figs. 14(a) and 14(b) for $\alpha=1.5 \pi$ and $\alpha=$ $1.7 \pi$ respectively. The fact that revivals are seen only at not very large rapidities (and the effect is stronger as closer $\psi$ to its minimal possible value) is surprising and contrasts to how causality is broken in the bulk. The structure of CTC is defined by $\alpha$ and $\psi$, and the time jumps become stronger as the angle defects and rapidities are increased. Thus we rather should expect that for high $\psi$ the time traveling along the CTC is more efficient in the sense that amplitudes of the classical free Green's function defined on the boundary are getting enhanced as $\alpha \psi$ grows. In the interacting holographic dual field theory the retarded Green's function is damped for larger $\alpha$ and $\psi$, so we can claim that causality in the boundary field theory is broken mildly as compared to the bulk.

Another interesting feature of this system is that while the overall weight of the Green function drastically decreases when the rapidity $\psi$ is taken away from the "forbidden region" on the diagram, the actual past time penetration depth [i.e. the deepest reachable point at negative times where $G^{\mathrm{cr}}(0,-\pi / 2 ; t,-\pi / 2) \neq 0$ ] increases (though very moderately), and this is in agreement with the "naive" intuition.

\section{CONCLUSIONS}

Under the assumption that the AdS/CFT correspondence is valid in the case of noncausal bulk geometry, we used its geodesic limit to calculate a two-point function of a $(1+1)$-dimensional field theory dual to the DeDeo-Gott time machine geometry, and demonstrated that the corresponding boundary propagator has remarkable features. We discovered that in the presence of closed timelike curves in the AdS bulk a causal propagation of an excitation from the future to the past is possible on the boundary, and the retarded Green function has a finite weight at negative times, exhibiting peaks at certain negative times. At positive times analytic structure of the Green function also changes, and new singularities arise due to nontrivial winding.

Surprisingly, we have found that as we increase the strength of the conical defects $\alpha$ and the rapidity $\psi$, the causality violation in the dual field theory is getting milder in the sense that the weight of the retarded Green's function at negative times decreases. 
Contra to the previous results on the dynamics of physical systems in time machine backgrounds [30-33], our calculations have demonstrated that sometimes it is possible to define evolution of an interacting theory in a time machine without imposing any additional selfconsistency constraints. Despite the explicit noncausality the Green's function calculated within the adopted approach does not seem to suffer from uncontrollable pathologies.

Our considerations leave a number of open questions. First of all, we have to understand how to interpret the boundary state dual to the DeDeo-Gott geometry, whether this quantum state is pathological or just an exotic yet physical state. From the boundary point of view a single conical defect, if its angular deficit is $\alpha=2 \pi(1-1 / N)$, can be thought of as state created by a nonlocal twist operator in a conformal field theory [41,47-51]. But what it means to have such an interplay of two independently boosted defects has to be clarified.

Another thing we have not touched on in the paper is the entanglement structure of the boundary state. We focused on the properties of the retarded Green function, and thus analyzed the timelike quasigeodesics. However, even below the $\alpha=\pi$ threshold, when the CTC are not present in the system, due to the lensing it is possible to connect timelike separated boundary points just by standard continuous spacelike geodesics. If a certain generalization of the Ryu-Takayanagi conjecture [52] is true in this case, it would mean the boundary state is timelike entangled [53]. To pursue the possible physical outcomes of this fact is an interesting direction for future research.

\section{ACKNOWLEDGMENTS}

We are grateful to Dmitry Ageev for collaboration during a certain stage of the project. It is our pleasure to thank Simon DeDeo, Mikhail Katsnelson, Joris Vanhoof, and Jan Zaanen for illuminating discussions. The work of I. A. is partially supported by RFBR Grant No. 14-01-00707. A. B. and K.S. are supported in part by the Netherlands Organization for Scientific Research/Ministry of Science and Education (NWO/OCW) and by the Foundation for Research into Fundamental Matter (FOM). K. S. is also supported by a VICI grant of the Netherlands Organization for Scientific Research (NWO).
[1] K. Godel, An example of a new type of cosmological solutions of Einstein's field equations of gravitation, Rev. Mod. Phys. 21, 447 (1949).

[2] W. J. van Stockum, The gravitational field of a distribution of particles rotating about an axis of symmetry, Proc. R. Soc. Edinburgh, Sect. A 57, 135 (1938).

[3] F. J. Tipler, Rotating cylinders and the possibility of global causality violation, Phys. Rev. D 9, 2203 (1974); Causality Violation in Asymptotically Flat Space-Times, Phys. Rev. Lett. 37, 879 (1976).

[4] M.S. Morris and K.S. Thorne, Wormholes in spacetime and their use for interstellar travel: A tool for teaching general relativity, Am. J. Phys. 56, 395 (1988).

[5] M. S. Morris, K. S. Thorne, and U. Yurtsever, Wormholes, Time Machines, and the Weak Energy Condition, Phys. Rev. Lett. 61, 1446 (1988).

[6] J. R. Gott III, Closed Timelike Curves Produced by Pairs of Moving Cosmic Strings: Exact Solutions, Phys. Rev. Lett. 66, 1126 (1991).

[7] A. Ori, Formation of closed timelike curves in a composite vacuum/dust asymptotically flat spacetime, Phys. Rev. D 76, 044002 (2007).

[8] F. S. N. Lobo, Time machines and traversable wormholes in modified theories of gravity, EPJ Web Conf. 58, 01006 (2013).

[9] O. Bertolami and R. Zambujal Ferreira, Wormholes and time-machines in nonminimally coupled mattercurvature theories of gravity, EPJ Web Conf. 58, 01008 (2013).
[10] S. M. Carroll, E. Farhi, and A. H. Guth, An Obstacle to Building a Time Machine, Phys. Rev. Lett. 68, 263 (1992); 68, 3368 (1992).

[11] S. Deser, R. Jackiw, and G. 't Hooft, Physical Cosmic Strings do not Generate Closed Timelike Curves, Phys. Rev. Lett. 68, 267 (1992).

[12] I. Y. Aref'eva and I. V. Volovich, The null energy condition and cosmology, Theor. Math. Phys. 155, 503 (2008).

[13] V. A. Rubakov, The null energy condition and its violation, Phys. Usp. 57, 128 (2014).

[14] P. Creminelli, A. Nicolis, and E. Trincherini, Galilean Genesis: An alternative to inflation, J. Cosmol. Astropart. Phys. 11 (2010) 021.

[15] P. Creminelli, M. A. Luty, A. Nicolis, and L. Senatore, Starting the Universe: Stable violation of the null energy condition and non-standard cosmologies, J. High Energy Phys. 12 (2006) 080.

[16] S. W. Hawking, Chronology protection conjecture, Phys. Rev. D 46, 603 (1992).

[17] D. Brace, C. A. R. Herdeiro, and S. Hirano, Classical and quantum strings in compactified pp waves and Godel type universes, Phys. Rev. D 69, 066010 (2004).

[18] M. S. Costa, C. A. R. Herdeiro, J. Penedones, and N. Sousa, Hagedorn transition and chronology protection in string theory, Nucl. Phys. B728, 148 (2005).

[19] J. P. Gauntlett and J. B. Gutowski, Supersymmetric solutions of minimal gauged supergravity in five dimensions, Phys. Rev. D 68, 105009 (2003); 70, 089901(E) (2004). 
[20] E. K. Boyda, S. Ganguli, P. Horava, and U. Varadarajan, Holographic protection of chronology in universes of the Godel type, Phys. Rev. D 67, 106003 (2003).

[21] E. G. Gimon and P. Horava, Over-rotating black holes, Godel holography and the hypertube, arXiv:hep-th/0405019.

[22] D. Brecher, P. A. DeBoer, D. C. Page, and M. Rozali, Closed timelike curves and holography in compact plane waves, J. High Energy Phys. 10 (2003) 031.

[23] C. A. R. Herdeiro, Spinning deformations of the D1-D5 system and a geometric resolution of closed timelike curves, Nucl. Phys. B665, 189 (2003).

[24] C. A. R. Herdeiro, Special properties of five-dimensional BPS rotating black holes, Nucl. Phys. B582, 363 (2000).

[25] M. M. Caldarelli, D. Klemm, and P. J. Silva, Chronology protection in anti-de Sitter, Classical Quantum Gravity 22, 3461 (2005).

[26] M. M. Caldarelli, Chronology protection in AdS/CFT, Fortschr. Phys. 54, 317 (2006).

[27] J. P. Gauntlett, J. B. Gutowski, and N. V. Suryanarayana, A deformation of $\operatorname{AdS}(5) \times S^{5}$, Classical Quantum Gravity 21, 5021 (2004).

[28] A. de la Fuente and R. Sundrum, Holography of the BTZ black hole, inside and out, J. High Energy Phys. 09 (2014) 073.

[29] I. Y. Arefeva, T. Ishiwatari, and I. V. Volovich, Cauchy problem on nonglobally hyperbolic spacetimes, Theor. Math. Phys. 157, 1646 (2008).

[30] O. V. Groshev, N. A. Gusev, E. A. Kuryanovich, and I. V. Volovich, On solutions to the wave equation on nonglobally hyperbolic manifold, Proc Steklov Inst Math / Trudy Matematicheskogo instituta imeni VA Steklova 265, 262275 (2009).

[31] F. Echeverria, G. Klinkhammer, and K. S. Thorne, Billiard balls in wormhole space-times with closed timelike curves: Classical theory, Phys. Rev. D 44, 1077 (1991).

[32] D. G. Boulware, Quantum field theory in spaces with closed timelike curves, Phys. Rev. D 46, 4421 (1992).

[33] J. L. Friedman, N. J. Papastamatiou, and J. Z. Simon, Failure of unitarity for interacting fields on space-times with closed timelike curves, Phys. Rev. D 46, 4456 (1992).

[34] T. G. Mackay and A. Lakhtakia, Towards a metamaterial simulation of a spinning cosmic string, Phys. Lett. A 374, 2305 (2010).

[35] M. Ringbauer, M. A. Broome, C. R. Myers, A. G. White, and T. C. Ralph, Experimental simulation of closed timelike curves, Nat. Commun. 5, 4145 (2014).

[36] S. DeDeo and J. R. Gott III, An eternal time machine in $(2+1)$-dimensional anti-de Sitter space, Phys. Rev. D 66, 084020 (2002); 67, 069902(E) (2003).
[37] S. Holst and H.J. Matschull, The anti-de Sitter Gott universe: A rotating BTZ wormhole, Classical Quantum Gravity 16, 3095 (1999).

[38] V. Balasubramanian and S. F. Ross, Holographic particle detection, Phys. Rev. D 61, 044007 (2000).

[39] V. Balasubramanian, A. Naqvi, and J. Simon, A multiboundary AdS orbifold and DLCQ holography: A universal holographic description of extremal black hole horizons, J. High Energy Phys. 08 (2004) 023.

[40] V. Balasubramanian, P. Kraus, and M. Shigemori, Massless black holes and black rings as effective geometries of the D1-D5 system, Classical Quantum Gravity 22, 4803 (2005).

[41] V. Balasubramanian, B. D. Chowdhury, B. Czech, and J. de Boer, Entwinement and the emergence of spacetime, J. High Energy Phys. 01 (2015) 048.

[42] V. Balasubramanian, A. Bernamonti, B. Craps, V. Keränen, E. Keski-Vakkuri, B. Müller, L. Thorlacius, and J. Vanhoof, Thermalization of the spectral function in strongly coupled two dimensional conformal field theories, J. High Energy Phys. 04 (2013) 069.

[43] P. Di Francesco, P. Mathieu, and D. Senechal, Conformal Field Theory (Springer-Verlag, New York, 1997), p. 890.

[44] M. Einsiedler and T. Ward, Geodesic Flow on Quotients of the Hyperbolic Plane, Ergodic Theory (Springer, London, 2011), pp. 277-330.

[45] V. Balasubramanian, B. Czech, V. E. Hubeny, K. Larjo, M. Rangamani, and J. Simon, Typicality versus thermality: An analytic distinction, Gen. Relativ. Gravit. 40, 1863 (2008).

[46] E. da Silva, E. Lopez, J. Mas, and A. Serantes, Collapse and revival in holographic quenches, J. High Energy Phys. 04 (2015) 038.

[47] O. Lunin and S.D. Mathur, Correlation functions for MN/SN orbifolds, Commun. Math. Phys. 219, 399 (2001).

[48] V. Balasubramanian, J. de Boer, E. Keski-Vakkuri, and S. F. Ross, Supersymmetric conical defects: Towards a string theoretic description of black hole formation, Phys. Rev. D 64, 064011 (2001).

[49] J. M. Maldacena and L. Maoz, Desingularization by rotation, J. High Energy Phys. 12 (2002) 055.

[50] E. J. Martinec and W. McElgin, String theory on AdS orbifolds, J. High Energy Phys. 04 (2002) 029.

[51] E. J. Martinec and W. McElgin, Exciting AdS orbifolds, J. High Energy Phys. 10 (2002) 050.

[52] S. Ryu and T. Takayanagi, Aspects of holographic entanglement entropy, J. High Energy Phys. 08 (2006) 045.

[53] S. J. Olson and T. C. Ralph, Entanglement Between the Future and Past in the Quantum Vacuum, Phys. Rev. Lett. 106, 110404 (2011). 